\title{
A virtual element generalization on polygonal meshes of the Scott-Vogelius finite element method for the 2-D Stokes problem
}

\author{
G. Manzini ${ }^{\mathrm{a}}$ and A. Mazzia ${ }^{\mathrm{b}}$ \\ ${ }^{a}$ T-5 Applied Mathematics and Plasma Physics Group, Los Alamos National Laboratory, Los Alamos, NM 87545, USA \\ ${ }^{\mathrm{b}}$ Dipartimento di Ingegneria Civile, Edile e Ambientale - ICEA, Università di Padova, 35131 Padova, Italy
}

\begin{abstract}
The Virtual Element Method (VEM) is a Galerkin approximation method that extends the Finite Element Method (FEM) to polytopal meshes. In this paper, we present a conforming formulation that generalizes the Scott-Vogelius finite element method for the numerical approximation of the Stokes problem to polygonal meshes in the framework of the virtual element method. In particular, we consider a straightforward application of the virtual element approximation space for scalar elliptic problems to the vector case and approximate the pressure variable through discontinuous polynomials. We assess the effectiveness of the numerical approximation by investigating the convergence on a manufactured solution problem and a set of representative polygonal meshes. We numerically show that this formulation is convergent with optimal convergence rates except for the lowest-order case on triangular meshes, where the method coincides with the $\mathbb{P}_{1}-\mathbb{P}_{0}$ Scott-Vogelius scheme, and on square meshes, which are situations that are well-known to be unstable.
\end{abstract}

Key words: Virtual Element Method, Stokes Equations, Scott-Vogelius finite element method

\section{Introduction}

Many physical phenomena can be described using the system of incompressible Stokes equations as, for example, sedimentation and bio-suspension processes [59], droplet dynamics [61], micro-fluidic devices [72], fibrous filter design [63] and Stokes flows in porous media [7]. The Finite Element Method (FEM) was proven to be very successful in the numerical treatment of the Stokes equations in variational form, see $[43,54,58]$. Although the FEM is very effective, it is also limited to a very specific kind of unstructured meshes. In fact, the Partial Differential Equations (PDEs) are discretized by suitable polynomial trial and test functions that are conveniently built only on unstructured meshes of triangular and quadrilateral elements in two-dimensions (2-D) and tetrahedral and hexahedral elements in three dimensions (3-D). In the last decades, a great effort has been spent to overcome this limitation and to develop numerical methods for steady state and time-dependent problems working on more general polygonal and polyhedral meshes, [21,62,73-77].

The Mimetic Finite Difference (MFD) method [21,64] was among the first effective approaches to be proposed in this direction. In fact, this numerical approach uses only the degrees of freedom without referring to any specific set of shape functions, and its design allows the numerical model to preserve several fundamental properties of the PDEs, such as maximum/minimum principles, solution symmetries and the conservation of mass, momentum and energy, see $[44,60]$. Originally proposed for the numerical approximation of diffusion problems $[40,41]$, the MFD method was then extended to convection-diffusion problems [48], and Stokes equations [18-20]. 
The variational reformulation of the MFD method led to the Virtual Element Method (VEM) [8]. The VEM is a Galerkin method such as the finite element method where the approximation space in every mesh element is composed by the solutions of a differential problem. The basis functions of such Galerkin formulation exist as specific solutions of the elemental problems and are uniquely identified by a special choice of the degrees of freedom. However, they are "virtual" as they are never built explicitly, and the bilinear forms of the variational formulation are approximated by using special polynomial projections that are computable from the degrees of freedom.

The VEM is strictly related to the finite element formulations on polygonal and polyhedral meshes $[49,55,65]$. Strong connections also exist with other discretization methods that work on such kind of meshes, as for example the discontinuous skeletal gradient discretizations [55], and the Boundary Element Method-based FEM (BEM-based FEM) [47].

The first virtual element method was formulated as a conforming FEM for the Poisson problem [8], and then extended to convection-reaction-diffusion problems with variable coefficients $[2,15]$. Similarly, the nonconforming formulation was proposed for the Poisson equation [6], and later extended to general elliptic problems [33,50], Stokes problem [46], eigenvalue problems [56], and the biharmonic equation [4,79]. The mixed virtual element method was proposed in [39] and [13] as the extension to the virtual element setting of the Brezzi-Douglas-Marini and RaviartThomas mixed methods [37]. The connection with the de Rham diagrams and the Nedelec elements and possible applications to electromagnetics have been explored in [12]. A (surely non-exhaustive) list of other significant applications of the VEM includes the works of References [3, 5,9-17,21-36,42,45, 51, 52, 67-70, 78].

In this work, we are interested in extending the Scott-Vogelius finite element method for the discretization of the 2D Stokes equation to the virtual element setting. The method that we present considers a discrete representation of the two components of the velocity field by using the conforming virtual element space originally proposed in [8,9] and its modified ("enhanced") version proposed in [2]. The scalar unknown, e.g., the pressure, is approximated by discontinuous polynomials on the mesh elements. The resulting discretization at the lowest order case on triangular meshes coincides with the $\mathbb{P}_{1}-\mathbb{P}_{0}$ Scott-Vogelius FEM. In such a case, the scheme can be non-convergent as the "infsup" stability condition cannot be ensured. However, in all other cases our VEM provides a different discretization, which performs well in the experiments we carried out. The zero divergence constraint is satisfied in a variational sense, i.e., the projection of the divergence on the subset of polynomials used in the scheme formulation is zero. It is worth mentioning that other virtual element approaches were recently proposed in the literature that approximate the Stokes velocity in such a way that its divergence is a polynomial that is set to zero in the scheme. This strategy provides an approximation of the Stokes velocity that satisfies the zero divergence constraint in a pointwise sense. We refer the interested reader to the works of References [17, 23, 24, 28, 53]. However, the polynomial projection of the velocity divergence in our VEM is zero up to the machine precision. If we consider such projection as our numerical approximation to the velocity divergence, such approximation is identically zero in the computational domain.

The paper is organized as follows. The Stokes equations in strong and variational form are introduced in Section 2. The virtual element method and its connection with the Scott-Vogelius finite element method are presented and discussed in Section 3. The convergence behavior of our VEM is established in Section 4, where we derive an error estimate for both velocity and pressure in the energy norm, and investigated numerically in Section 5 through a manufactured solution test case that is solved on a set of representative polygonal meshes. This set of meshes includes also the triangular meshes where the low-order original Scott-Vogelius finite element method may show a well-known unstable behavior, thus motivating us to study the "inf-sup" stability of the method numerically. Final remarks and hints for future work are given in Section 6.

\section{The Stokes problem and the virtual element discretization}

\subsection{Notation and technicalities}

Consider the integer number $k>0$ and let $\omega$ be a bounded, open, connected subset of $\mathbb{R}^{2}$. According to the notation and definitions given in [1], $L^{2}(\omega)$ is the linear space of square integrable functions defined on $\omega$ and $H^{k}(\omega)$, is the linear subspace of functions in $L^{2}(\omega)$ whose weak derivatives of order less than or equal to $k$ are also in $L^{2}(\omega)$. We denote the norm and seminorm in $H^{k}(\omega)$ by $\|\cdot\|_{k, \omega}$ and $|\cdot|_{k, \omega}$, respectively. Throughout the paper, we prefer 
denoting the scalar product between scalar and vector-valued fields by the integral notation, even if sometimes we use the notation " $(\cdot, \cdot)$ " for the sake of conciseness.

In the formulation of the Stokes problem on the computational domain $\Omega$, we use the linear space

$$
L_{0}^{2}(\Omega):=\left\{q \in L^{2}(\Omega): \int_{\Omega} q d \mathbf{x}=0\right\},
$$

which is clearly a subspace of $L^{2}(\Omega)$. This subspace is isomorphic to the quotient space $L^{2}(\Omega) \backslash \mathbb{R}$, where two square integrable functions are equivalent and identified as members of the same equivalence class (which we still call "function") if their difference is constant.

\subsection{Strong and weak form of the Stokes problem}

We are interested in the numerical discretization of the system of incompressible Stokes equations

$$
\begin{aligned}
& -\Delta \mathbf{u}+\nabla p=\mathbf{f} \quad \text { in } \Omega, \\
& \operatorname{div} \mathbf{u}=0 \quad \text { in } \Omega,
\end{aligned}
$$

for the velocity vector $\mathbf{u}$ and the pressure $p$ defined on the computational domain $\Omega$, which we assume to be an open, bounded, polygonal subset of $\mathbb{R}^{2}$, whose boundary is denoted by $\Gamma$. For the mathematical well-posedness of this problem, we consider the homogeneous Dirichlet boundary condition

$$
\mathbf{u}=0 \quad \text { on } \Gamma .
$$

Assumption (4) makes the exposition simpler and immediate and is not restrictive on the design of the scheme as more general boundary conditions can be included with some additional technicalities. In particular, including nonhomogeneous Dirichlet boundary conditions is straightforward and this case will be considered in the numerical experiments.

To write the variational formulation of problem (2)-(4), we introduce the bilinear forms

$$
a(\cdot, \cdot):\left[H^{1}(\Omega)\right]^{2} \times\left[H^{1}(\Omega)\right]^{2} \rightarrow \mathbb{R}
$$

and

which are defined as:

$$
b(\cdot, \cdot):\left[H^{1}(\Omega)\right]^{2} \times L^{2}(\Omega) \rightarrow \mathbb{R},
$$

$$
\begin{aligned}
a(\mathbf{v}, \mathbf{w}):=\int_{\Omega} \nabla \mathbf{v}: \nabla \mathbf{w} d \mathbf{x} & \forall \mathbf{v}, \mathbf{w} \in\left[H^{1}(\Omega)\right]^{2}, \\
b(\mathbf{v}, q):=-\int_{\Omega} q \operatorname{div} \mathbf{v} d \mathbf{x} & \forall \mathbf{v} \in\left[H^{1}(\Omega)\right]^{2}, q \in L^{2}(\Omega) .
\end{aligned}
$$

The symbol ":" in (5) is the standard "dot" product between two-dimensional tensors. Now, the variational formulation is given by: Find $(\mathbf{u}, p) \in\left[H_{0}^{1}(\Omega)\right]^{2} \times L_{0}^{2}(\Omega)$ such that

$$
\begin{aligned}
a(\mathbf{u}, \mathbf{v})+b(\mathbf{v}, p) & =(\mathbf{f}, \mathbf{v}) & & \forall \mathbf{v} \in\left[H_{0}^{1}(\Omega)\right]^{2}, \\
b(\mathbf{u}, q) & =0 & & \forall q \in L_{0}^{2}(\Omega) .
\end{aligned}
$$

The existence and uniqueness of the solution pair $(\mathbf{u}, p)$, cf. [37,57,58], follow on noting that the bilinear form $a(\cdot, \cdot)$ is continuous and coercive, and the bilinear form $b(\cdot, \cdot)$ is continuous and satisfies the inf-sup condition:

$$
\inf _{q \in L_{0}^{2}(\Omega) \backslash \mathbb{R}} \sup _{\mathbf{v} \in\left[H_{0}^{1}(\Omega) \backslash \mathbb{R}\right]^{2}} \frac{b(\mathbf{v}, q)}{\|\mathbf{v}\|_{1, \Omega}\|q\|_{0, \Omega}} \geq \widehat{\beta},
$$

for some real, strictly positive constant $\widehat{\beta}$.

We consider the two finite-dimensional approximation spaces $\mathbf{V}_{k}^{h}$ and $Q_{k}^{h}$ for the vector and the scalar unknowns, where $k$ and $\underline{k}$ are two integer numbers such that $\underline{k} \leq k-1$. These spaces are labeled by $h$ to indicate that they are built on a given mesh $\Omega_{h}$. We assume that $\mathbf{V}_{k}^{h}$ is a conforming subspace of $\left[H_{0}^{1}(\Omega)\right]^{2}$ and $Q_{\underline{k}}^{h}$ a discontinuous subspace 
of $L_{0}^{2}(\Omega)$. We search for a vector field $\mathbf{u}_{h} \in \mathbf{V}_{k}^{h}$ and a scalar field $p_{h} \in Q_{\underline{k}}^{h}$ that approximate $\mathbf{u}$ and $p$, respectively. These fields are the solution of the following variational problem: Find $\left(\mathbf{u}_{h}, p_{h}\right) \in \mathbf{V}_{k}^{h} \times Q_{\underline{k}}^{h}$ such that

$$
\begin{aligned}
a_{h}\left(\mathbf{u}_{h}, \mathbf{v}_{h}\right)+b_{h}\left(\mathbf{v}_{h}, p_{h}\right) & =\left\langle\mathbf{f}_{h}, \mathbf{v}_{h}\right\rangle & & \forall \mathbf{v}_{h} \in \mathbf{V}_{k}^{h}, \\
b_{h}\left(\mathbf{u}_{h}, q_{h}\right) & =0 & & \forall q_{h} \in Q_{\underline{k}}^{h} .
\end{aligned}
$$

In equations (10) and (11) we use the virtual element approximation of the bilinear forms $a(\cdot, \cdot)$ and $b(\cdot, \cdot)$, which are denoted by $a_{h}(\cdot, \cdot): \mathbf{V}_{k}^{h} \times \mathbf{V}_{k}^{h} \rightarrow \mathbb{R}$ and $b_{h}(\cdot, \cdot): \mathbf{V}_{k}^{h} \times Q_{k}^{h} \rightarrow \mathbb{R}$, respectively. Similarly, in the right-hand side of equation (10) we use the virtual element approximation of the right-hand side of (7), here denoted by $\left\langle\mathbf{f}_{h}, \cdot\right\rangle$, where $\mathbf{f}_{h}$ is assumed to be an element of the dual space of $\mathbf{V}_{k}^{h}$.

\section{The virtual element generalization of the Scott-Vogelius finite element method}

\subsection{Mesh definition and regularity assumptions}

Let $\mathcal{T}=\left\{\Omega_{h}\right\}_{h}$ be a mesh family for $\Omega$, where every mesh $\Omega_{h}$ is a finite set of (closed) polygonal elements E such that $\bar{\Omega}=\cup_{\mathrm{E} \in \Omega_{h}}$ E. Each mesh is labeled by the subindex $h$, which is, as usual, the maximum of the diameters of the mesh elements, i.e., $h_{\mathrm{E}}=\sup _{\mathbf{x}, \mathbf{y} \in \mathrm{E}}|\mathbf{x}-\mathbf{y}|$. We assume that all the elements of a given mesh are nonoverlapping in the sense that the intersection of the closure in $\mathbb{R}^{2}$ of any pair of them can only be a mesh vertex or a mesh edge. Therefore, the area of such intersection is zero. Every polygonal element $\mathrm{E}$ has $N_{\mathrm{E}}^{\mathcal{V}}$ vertices with coordinates $\mathbf{x}_{\mathrm{v}}=\left(x_{\mathrm{v}}, y_{\mathrm{v}}\right)$. These vertices are connected by $N_{\mathrm{E}}^{\mathcal{E}}$ nonintersecting straight edges e, which form the boundary $\partial \mathrm{E}$. The measure of $\mathrm{E}$ is denoted by $|\mathrm{E}|$, its centroid (e.g., the barycenter) by $\mathbf{x}_{\mathrm{E}}:=\left(x_{\mathrm{E}}, y_{\mathrm{E}}\right)$, the unit outward vector orthogonal to the elemental edge e by $\mathbf{n}_{\mathrm{E}, \mathrm{e}}$. We also introduce the unit vector $\mathbf{n}_{\mathrm{e}}$, which is orthogonal to the edge $\mathrm{e}$ and whose orientation is independent of the elements E, but it is fixed once and for all in the mesh. Note that for any given edge e, the vector $\mathbf{n}_{\mathrm{E}, \mathrm{e}}$ may differ from $\mathbf{n}_{\mathrm{e}}$ only by the multiplicative factor -1 .

The mesh sequence used in the formulation of the method is required to satisfy the two following conditions: there exists a positive constant $\varrho$ such that

- (M1) all polygonal elements E are star-shaped with respect to a disk with a radius, $r$, such that $r \geq \varrho h_{\mathrm{E}}$;

- (M2) all polygonal edges e $\in \partial \mathrm{E}$ of all polygonal elements $\mathrm{E}$ satisfy $h_{\mathrm{e}} \geq \varrho h_{\mathrm{E}}$, where $h_{\mathrm{e}}$ is the edge length.

We assume that $\varrho$ is independent of $h$, so that these regularity assumptions are uniformly satisfied by all the meshes $\Omega_{h}$ of the mesh family $\mathcal{T}=\left\{\Omega_{h}\right\}_{h}$ used in the VEM formulation. Property (M1) implies that all the mesh elements are simply connected subsets of $\mathbb{R}^{2}$. Property (M2) implies that the number of edges in the elemental boundaries is uniformly bounded over the whole mesh family $\mathcal{T}$. We remark that under these conditions the theory of polynomial approximation of functions in Sobolev spaces [38] provides interpolation and projection error estimates that can be used in the analysis of the method, see, e.g., Section 4.1.

\subsection{Scalar and vector approximation spaces}

If $\mathrm{E}$ is a generic mesh element and $\ell$ a nonnegative integer number, we let $\mathbb{P}_{\ell}(\mathrm{E})$ denote the linear space of polynomials of degree up to $\ell$ defined on $E$, with the useful convention that $\mathbb{P}_{-1}(E)=\{0\}$, and $\left[\mathbb{P}_{\ell}(E)\right]^{2}$ denotes the space of two-dimensional vector polynomials of degree up to $\ell$ on $\mathrm{E}$. We use the notation $\mathbb{P}_{\ell}\left(\Omega_{h}\right)$ for the elementwise polynomials of degree $\ell$ defined on the mesh $\Omega_{h}$, so that $q \in \mathbb{P}_{\ell}\left(\Omega_{h}\right)$ is such that $q_{\text {IE }} \in \mathbb{P}_{\ell}(\mathrm{E})$ for all E $\in \Omega_{h}$. Similarly, if e is a generic mesh edge, we let $\mathbb{P}_{\ell}(\mathrm{e})$ denote the linear space of polynomials of degree up to $\ell$ defined on e.

For the approximation of the scalar unknown $p$, we consider the functional space of discontinuous polynomials of degree $\underline{k}$ having zero average on the mesh $\Omega_{h}$, which we formally define as $Q_{\underline{k}}^{h}:=\mathbb{P}_{\underline{k}}\left(\Omega_{h}\right) \cap L_{0}^{2}(\Omega)$.

Instead, for the approximation of the vector unknown $\mathbf{u}$ we consider the functional space of vector-valued functions

$$
\mathbf{V}_{k}^{h}:=\left\{\mathbf{v}_{h} \in\left[H_{0}^{1}(\Omega)\right]^{2}: \mathbf{v}_{h \mid \mathrm{E}} \in \mathbf{V}_{k}^{h}(\mathrm{E}) \quad \forall \mathrm{E} \in \Omega_{h}\right\}, \quad k \geq 1,
$$



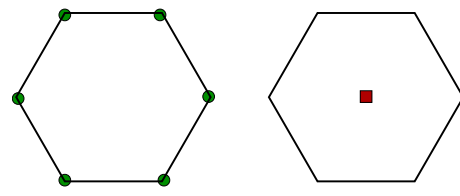

$(\mathbf{k}, \underline{\mathbf{k}})=(\mathbf{1}, \mathbf{0})$
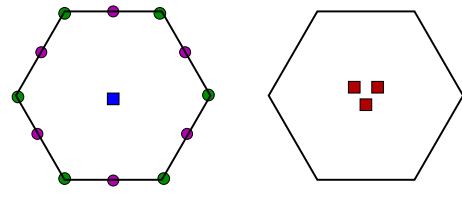

$(\mathbf{k}, \underline{\mathbf{k}})=(\mathbf{2}, \mathbf{1})$
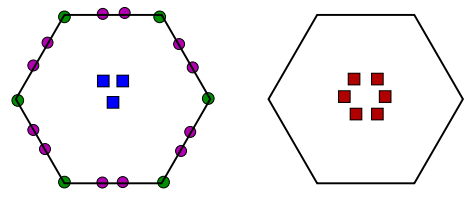

$(\mathbf{k}, \underline{\mathbf{k}})=(3,2)$

Fig. 1. Degrees of freedom of each component of the virtual element vector-valued fields of $\mathbf{V}_{k}^{h}(\mathrm{E})$ (left) and the scalar polynomial fields of $Q_{k}^{h}(\mathrm{E})$ (right) of an hexagonal element for the accuracy degrees $k=1,2,3$ and $\underline{k}=k-1$. Vertex values and edge polynomial moments are marked by a circular bullet; cell polynomial moments are marked by a square bullet.

which is a conforming finite-dimensional subspace of $\left[H_{0}^{1}(\Omega)\right]^{2}$. This functional space is obtained by "gluing together" the local virtual element spaces $\mathbf{V}_{k}^{h}(\mathrm{E})$ defined on the mesh elements $\mathrm{E} \in \Omega_{h}$. We take $\mathbf{V}_{k}^{h}(\mathrm{E})=\left[V_{k}^{h}(\mathrm{E})\right]^{2}$ where $V_{k}^{h}(\mathrm{E})$ is either the scalar virtual element space originally introduced in [8]

$$
V_{k}^{h}(\mathrm{E}):=\left\{v_{h} \in H^{1}(\mathrm{E}): v_{h \mid \partial \mathrm{E}} \in C^{0}(\partial \mathrm{E}), v_{h \mid \mathrm{e}} \in \mathbb{P}_{k}(\mathrm{e}) \forall \mathrm{e} \in \partial \mathrm{E}, \Delta v_{h} \in \mathbb{P}_{k-2}(\mathrm{E})\right\},
$$

or its "modified" version (also called "enhanced" in the virtual element literature) given by [2]

$$
\begin{array}{r}
V_{k}^{h}(\mathrm{E}):=\left\{v_{h} \in H^{1}(\mathrm{E}): v_{h \mid \partial \mathrm{E}} \in C^{0}(\partial \mathrm{E}), v_{h \mid \mathrm{e}} \in \mathbb{P}_{k}(\mathrm{e}) \forall \mathrm{e} \in \partial \mathrm{E}, \Delta v_{h} \in \mathbb{P}_{k}(\mathrm{E}),\right. \\
\left.\int_{\mathrm{E}}\left(v_{h}-\Pi_{k}^{\nabla, \mathrm{E}} v_{h}\right) q d \mathbf{x}=0 \quad \forall q \in \mathbb{P}_{k}(\mathrm{E}) \backslash \mathbb{P}_{k-2}(\mathrm{E})\right\},
\end{array}
$$

where $\mathbb{P}_{k}(\mathrm{E}) \backslash \mathbb{P}_{k-2}(\mathrm{E})$ is the space of polynomials of degree exactly equal to $k$ or $k-1$ and $\Pi_{k}^{\nabla, \mathrm{E}}$ is the elliptic projection operator that will be defined in Section 3.3, cf. equations (19)-(20). To avoid the proliferation of symbols practically denoting the same thing, we use the notation " $V_{k}^{h}$ " for the spaces in (13) and (14) with a minor abuse of notation. The definition of the virtual element vector space in (14) has been modified according to the so-called enhancement strategy, so that the $L^{2}$-orthogonal projection onto $\left[\mathbb{P}_{k}(\mathrm{E})\right]^{2}$ of a virtual element function $\mathbf{v}_{h} \in \mathbf{V}_{k}^{h}(\mathrm{E})$ is computable.

Remark 3.1 (Connection with the Scott-Vogelius finite element method) The approximation spaces defined above contain the Scott-Vogelius finite element method as a special case. In fact, if we consider a triangular mesh, we recover the Scott-Vogelius FEM by setting $\mathbf{V}_{k}^{h}=\mathbf{V}_{k}^{S V, h}$, where

$$
\begin{aligned}
\mathbf{V}_{k}^{S V, h} & :=\left\{\mathbf{v}_{h} \in\left[H^{1}(\Omega)\right]^{2}: \mathbf{v}_{h \mid E} \in\left[\mathbb{P}_{k}(E)\right]^{2} \quad \forall E \in \Omega_{h}\right\}, \\
Q_{\underline{k}}^{h} & :=\left\{q_{h} \in L_{0}^{2}(\Omega): q_{h \mid E} \in \mathbb{P}_{\underline{k}}(E) \quad \forall E \in \Omega_{h}\right\}=\mathbb{P}_{\underline{k}}\left(\Omega_{h}\right) \cap L_{0}^{2}(\Omega),
\end{aligned}
$$

for $k \geq 1$ and $\underline{k} \leq k-1$, and taking $a_{h}(\cdot, \cdot)=a(\cdot, \cdot), b_{h}(\cdot, \cdot)=b(\cdot, \cdot)$ in (10)-(11). It is well-known that the scheme with $k=1$ and $\underline{k}=0$ can be unstable on triangular and square meshes. It is worth noting that our virtual element method coincides with such approximations only on triangular and square meshes and only for $k=1$ and $\underline{k}=0$. In all the other cases, including for example $k=2$ and $\underline{k}=0$, our approach provides a different and well-behaving discretization. The performance of the VEM for different values of the integer pair $(k, \underline{k})$ on triangular and square meshes is investigated in the numerical section. Our numerical evidence shows that the VEM is stable and convergent except for the possibly unstable cases mentioned above.

\subsection{Degrees of freedom and polynomial projection operators}

The functions in the virtual element space (13) and the enhanced space (14) are uniquely characterized by the following set of values, the degrees of freedom:

(D1) for $k \geq 1$, the vertex values $v_{h}\left(\mathbf{x}_{\mathrm{v}}\right), \mathrm{v} \in \partial \mathrm{E}$; 
(D2) for $k \geq 2$, the polynomial edge moments

$$
\frac{1}{|\mathrm{e}|} \int_{\mathrm{e}} v_{h}(s) q_{h}(s) d s \quad \forall q_{h} \in \text { basis of } \mathbb{P}_{k-2}(\mathrm{e})
$$

for every edge e $\in \partial \mathrm{E}$;

(D3) for $k \geq 2$, the polynomial cell moments

$$
\frac{1}{|\mathrm{E}|} \int_{\mathrm{E}} v_{h}(\mathbf{x}) q_{h}(\mathbf{x}) d \mathbf{x} \quad \forall q_{h} \in \text { basis of } \mathbb{P}_{k-2}(\mathrm{E}) .
$$

The unisolvence of (D1)-(D3) is proved in Reference [8] for the space defined in (13), and in Reference [2] for the enhanced space defined in (14). In Figure 1, we illustrate the degrees of freedom for each velocity component and the scalar field for the polynomial degree $k=1,2,3$ on an hexagonal element. The degrees of freedom that uniquely characterize the virtual element functions in the global space $\mathbf{V}_{k}^{h}$ are obtained by collecting the elemental degrees of freedom. Their unisolvence in the global space $\mathbf{V}_{k}^{h}$ is an immediate consequence of the unisolvence of the degrees of freedom (D1)-(D3) in the elemental spaces $\mathbf{V}_{k}^{h}(\mathrm{E})$.

Remark 3.2 In our implementation we used the scaled monomials and the orthogonal polynomials as two alternative bases in the definitions of the moments on $e \in \partial E$ and $E$ of the degrees of freedom (D2) and (D3). We remark that the choice of the basis of the polynomial space is arbitrary and does not change the theoretical convergence property of the method although it may impact on the conditioning of the discrete problem and practically affects the accuracy of the resulting approximation.

Remark 3.3 (Alternative choice of the degrees of freedom) Instead of (D2), we can select the values at a set of $k-1$ internal nodes on each edge, e.g., the nodes supporting the one-dimensional Gauss-Lobatto integration rule with $k-1$ internal nodes, which is exact on univariate polynomials of degree up to $2 k+1$. This alternative choice is equivalent to (D2). In the preliminary stages of our work, we carried out all numerical experiments with both choices of degrees of freedom and we did not find any significant difference in the results.

Let $v_{h}$ be a scalar virtual element function on the element $\mathrm{E}$ according to the definition given in (13) or (14). Then, the following polynomial projections are computable using the degrees of freedom (D1)-(D3):

- the elliptic projection $\Pi_{k}^{\nabla, \mathrm{E}} v_{h} \in \mathbb{P}_{k}(\mathrm{E})$, which is the solution of the variational problem

$$
\begin{aligned}
\int_{\mathrm{E}} \nabla\left(v_{h}-\Pi_{k}^{\nabla, \mathrm{E}} v_{h}\right) q d \mathbf{x} & =0 \quad \forall q \in \mathbb{P}_{k}(\mathrm{E}), \\
\int_{\partial \mathrm{E}}\left(v_{h}-\Pi_{k}^{\nabla, \mathrm{E}} v_{h}\right) d s & =0 ;
\end{aligned}
$$

- the orthogonal projection $\Pi_{\bar{k}}^{0, \mathrm{E}} v_{h} \in \mathbb{P}_{\bar{k}}(\mathrm{E})$, which is the solution of the variational problem

$$
\int_{\mathrm{E}}\left(v_{h}-\Pi_{\bar{k}}^{0, \mathrm{E}} v_{h}\right) q d \mathbf{x}=0 \quad \forall q \in \mathbb{P}_{\bar{k}}(\mathrm{E}),
$$

where $\bar{k}=k-2$ for $k \geq 2$ if $V_{k}^{h}(\mathrm{E})$ is defined by (13), and $\bar{k}=k \geq 1$ if $V_{k}^{h}(\mathrm{E})$ is defined by (14).

Likewise, if $\mathbf{v}_{h}=\left(v_{x}, v_{y}\right)^{T}$ is a vector-valued field whose components are in the virtual element spaces defined by (13) or (14), the following projectors are computable:

- the orthogonal projection $\Pi_{\bar{k}}^{0, \mathrm{E}} \mathbf{v}_{h}$ of a vector-valued field $\mathbf{v}_{h}=\left(v_{x}, v_{y}\right)^{T}$, which is the solution of the variational problem

$$
\int_{\mathrm{E}}\left(\mathbf{v}_{h}-\Pi_{\bar{k}}^{0, \mathrm{E}} \mathbf{v}_{h}\right) \cdot \mathbf{q} d \mathbf{x}=0 \quad \forall \mathbf{q} \in\left[\mathbb{P}_{\bar{k}}(\mathrm{E})\right]^{2},
$$

where $\bar{k}=k-2$ for $k \geq 2$ if each component of $\mathbf{v}_{h}$ belongs to the scalar virtual element space $V_{k}^{h}(\mathrm{E})$ defined by (13), and $\bar{k}=k \geq 1$ if each component of $\mathbf{v}_{h}$ belongs to the enhanced virtual element space $V_{k}^{h}(\mathrm{E})$ defined by (14). The projection operator is computed component-wisely, i.e., $\Pi_{\bar{k}}^{0, \mathrm{E}} \mathbf{v}_{h}=\left(\Pi_{\bar{k}}^{0, \mathrm{E}} v_{x}, \Pi_{\bar{k}}^{0, \mathrm{E}} v_{y}\right)^{T} \in\left[\mathbb{P}_{\bar{k}}(\mathrm{E})\right]^{2}$, where $\Pi_{\bar{k}}^{0, \mathrm{E}} v_{x}$ and $\Pi_{\bar{k}}^{0, \mathrm{E}} v_{y}$ are the scalar orthogonal projections defined above; 
- the orthogonal projection $\Pi_{k-1}^{0, \mathrm{E}} \nabla \mathbf{v}_{h} \in\left[\mathbb{P}_{k-1}(\mathrm{E})\right]^{2 \times 2}$ of $\nabla \mathbf{v}_{h}$, the gradient of the virtual element vector-valued field $\mathbf{v}_{h}$, onto the linear space of the $2 \times 2$-sized matrix-valued polynomials of degree $k-1$. These quantities are defined component-wisely as follows:

$$
\nabla \mathbf{v}_{h}=\left(\begin{array}{ll}
\frac{\partial v_{x}}{\partial x} & \frac{\partial v_{x}}{\partial y} \\
\frac{\partial v_{y}}{\partial x} & \frac{\partial v_{y}}{\partial y}
\end{array}\right)
$$

and

$$
\Pi_{k-1}^{0, \mathrm{E}} \nabla \mathbf{v}_{h}=\left(\begin{array}{cc}
\Pi_{k-1}^{0, \mathrm{E}} \frac{\partial v_{x}}{\partial x} & \Pi_{k-1}^{0, \mathrm{E}} \frac{\partial v_{x}}{\partial y} \\
\Pi_{k-1}^{0, \mathrm{E}} \frac{\partial v_{y}}{\partial x} & \Pi_{k-1}^{0, \mathrm{E}} \frac{\partial v_{y}}{\partial y}
\end{array}\right),
$$

and this latter one is the solution of the variational problem:

$$
\int_{\mathrm{E}}\left(\nabla \mathbf{v}_{h}-\Pi_{k-1}^{0, \mathrm{E}} \nabla \mathbf{v}_{h}\right): \boldsymbol{\kappa} d \mathbf{x}=0 \quad \forall \boldsymbol{\kappa} \in\left[\mathbb{P}_{k-1}(\mathrm{E})\right]^{2 \times 2} .
$$

The computability of the scalar polynomial projection is proved in $[2,8]$, while the computability of the vector polynomial projection can easily be proved by using the same arguments of these papers component-wisely.

\subsection{The virtual element bilinear forms $a_{h}(\cdot, \cdot)$}

First, we note that the linearity of the integral in (5) allows us to split the bilinear form $a(\cdot, \cdot)$ as a summation of the local bilinear forms defined on the mesh elements:

$$
a(\mathbf{v}, \mathbf{w})=\sum_{\mathrm{E} \in \Omega_{h}} a^{\mathrm{E}}(\mathbf{v}, \mathbf{w}) \quad \text { with } \quad a^{\mathrm{E}}(\mathbf{v}, \mathbf{w})=\int_{\mathrm{E}} \nabla \mathbf{v}: \nabla \mathbf{w} d \mathbf{x} .
$$

The virtual element bilinear form $a_{h}(\cdot, \cdot)$ can be defined as the sum of the local bilinear forms $a_{h}^{\mathrm{E}}(\cdot, \cdot): \mathbf{V}_{k}^{h}(\mathrm{E}) \times$ $\mathbf{V}_{k}^{h}(\mathrm{E}) \rightarrow \mathbb{R}$ by using the orthogonal projection operators:

$$
a_{h}\left(\mathbf{v}_{h}, \mathbf{w}_{h}\right)=\sum_{\mathrm{E} \in \Omega_{h}} a_{h}^{\mathrm{E}}\left(\mathbf{v}_{h}, \mathbf{w}_{h}\right)
$$

where

$$
a_{h}^{\mathrm{E}}\left(\mathbf{v}_{h}, \mathbf{w}_{h}\right)=\int_{\mathrm{E}} \Pi_{k-1}^{0, \mathrm{E}} \nabla \mathbf{v}_{h}: \Pi_{k-1}^{0, \mathrm{E}} \nabla \mathbf{w}_{h} d \mathbf{x}+S_{h}\left(\left(1-\Pi_{k}^{\mathrm{E}}\right) \mathbf{v}_{h},\left(1-\Pi_{k}^{\mathrm{E}}\right) \mathbf{w}_{h}\right),
$$

where $\Pi_{k}^{\mathrm{E}}$ in the last term can be either the orthogonal or the elliptic projection $\Pi_{k}^{0, \mathrm{E}}$ or $\Pi_{k}^{\nabla, \mathrm{E}}$. The definition in (25) includes the stabilization term provided by the local bilinear form $S_{h}(\cdot, \cdot): \mathbf{V}_{k}^{h}(\mathrm{E}) \times \mathbf{V}_{k}^{h}(\mathrm{E}) \rightarrow \mathbb{R}$. According to the virtual element setting, for $S_{h}(\cdot, \cdot)$ we can choose any symmetric, positive definite bilinear form such that

$$
\sigma_{*} a\left(\mathbf{v}_{h}, \mathbf{v}_{h}\right) \leq S_{h}\left(\mathbf{v}_{h}, \mathbf{v}_{h}\right) \leq \sigma^{*} a\left(\mathbf{v}_{h}, \mathbf{v}_{h}\right) \quad \forall \mathbf{v}_{h} \in \mathbf{V}_{k}^{h}(\mathrm{E}) \cap \operatorname{ker}\left(\Pi_{k}^{\mathrm{E}}\right),
$$

where $\sigma_{*}$ and $\sigma^{*}$ are two real, positive constants independent of $h$. A detailed study of possible stabilization forms can be found in [66]. This design is such that the following two fundamental properties are true for the local bilinear form $a_{h}^{\mathrm{E}}(\cdot, \cdot)$ :

- Stability: there exist two real, positive constants $\alpha_{*}$ and $\alpha^{*}$ such that

$$
\alpha_{*} a^{\mathrm{E}}\left(\mathbf{v}_{h}, \mathbf{v}_{h}\right) \leq a_{h}^{\mathrm{E}}\left(\mathbf{v}_{h}, \mathbf{v}_{h}\right) \leq \alpha^{*} a^{\mathrm{E}}\left(\mathbf{v}_{h}, \mathbf{v}_{h}\right) \quad \forall \mathbf{v}_{h} \in \mathbf{V}_{k}^{h}(\mathrm{E}) .
$$

These constants are independent of $h$ but can depend on other parameters of the discretization, such as $\rho$, which is related to the regularity of the mesh, $\sigma_{*}$ and $\sigma^{*}$, and the order of the method $k$. 
- Polynomial consistency: the exactness property holds

$$
a_{h}^{\mathrm{E}}\left(\mathbf{v}_{h}, \mathbf{q}\right)=a^{\mathrm{E}}(\mathbf{v}, \mathbf{q}),
$$

for every vector field $\mathbf{v}_{h} \in \mathbf{V}_{k}^{h}(\mathrm{E})$ and vector polynomial field $\mathbf{q} \in\left[\mathbb{P}_{k}(\mathrm{E})\right]^{2}$.

These properties have two important consequences. First, adding all the local left inequalities in (26) implies that the bilinear form $a_{h}(\cdot, \cdot)$ is coercive on $\mathbf{V}_{k}^{h} \times \mathbf{V}_{k}^{h}$ :

$$
a_{h}\left(\mathbf{v}_{h}, \mathbf{v}_{h}\right) \geq \alpha_{*}\left|\mathbf{v}_{h}\right|_{1, \Omega}^{2}
$$

Second, the local bilinear form $a_{h}^{\mathrm{E}}(\cdot, \cdot)$ is continuous on $\mathbf{V}_{k}^{h} \times \mathbf{V}_{k}^{h}$. Indeed, relation (26) and the symmetry of $a_{h}^{\mathrm{E}}(\cdot, \cdot)$ imply that $a_{h}^{\mathrm{E}}(\cdot, \cdot)$ is a semi-inner product on $\mathbf{V}_{k}^{h}(\mathrm{E})$ (indeed, it is an inner product on the quotient space $\left[V_{k}^{h}(\mathrm{E}) \backslash \mathbb{R}\right]^{2}$ ). Using the Cauchy-Schwarz inequality we find that:

$$
\begin{aligned}
a_{h}^{\mathrm{E}}\left(\mathbf{v}_{h}, \mathbf{w}_{h}\right) & \leq\left[a_{h}^{\mathrm{E}}\left(\mathbf{v}_{h}, \mathbf{v}_{h}\right)\right]^{\frac{1}{2}}\left[a_{h}^{\mathrm{E}}\left(\mathbf{w}_{h}, \mathbf{w}_{h}\right)\right]^{\frac{1}{2}} \leq \alpha^{*}\left[a^{\mathrm{E}}\left(\mathbf{v}_{h}, \mathbf{v}_{h}\right)\right]^{\frac{1}{2}}\left[a^{\mathrm{E}}\left(\mathbf{w}_{h}, \mathbf{w}_{h}\right)\right]^{\frac{1}{2}} \\
& =\alpha^{*}\left|\mathbf{v}_{h}\right|_{1, \mathrm{E}}\left|\mathbf{w}_{h}\right|_{1, \mathrm{E}} .
\end{aligned}
$$

Then, we sum all the local inequalities and use again the Cauchy-Schwarz inequality and the right inequality of (26) to find that

$$
\begin{aligned}
a_{h}\left(\mathbf{v}_{h}, \mathbf{w}_{h}\right) & =\sum_{\mathrm{E} \in \Omega_{h}} a_{h}^{\mathrm{E}}\left(\mathbf{v}_{h}, \mathbf{w}_{h}\right) \leq \alpha^{*} \sum_{\mathrm{E} \in \Omega_{h}}\left|\mathbf{v}_{h}\right|_{1, \mathrm{E}}\left|\mathbf{w}_{h}\right|_{1, \mathrm{E}} \\
& \leq \alpha^{*}\left(\sum_{\mathrm{E} \in \Omega_{h}}\left|\mathbf{v}_{h}\right|_{1, \mathrm{E}}^{2}\right)^{\frac{1}{2}}\left(\sum_{\mathrm{E} \in \Omega_{h}}\left|\mathbf{w}_{h}\right|_{1, \mathrm{E}}^{2}\right)^{\frac{1}{2}}=\alpha^{*}\left|\mathbf{v}_{h}\right|_{1, \Omega}\left|\mathbf{w}_{h}\right|_{1, \Omega},
\end{aligned}
$$

which implies the global continuity of $a_{h}$.

\subsection{The virtual element bilinear forms $b_{h}(\cdot, \cdot)$}

First, we note that the linearity of the integral in (6) allows us to split the bilinear form $b(\cdot, \cdot)$ as a summation of the local bilinear forms defined on the mesh elements:

$$
b(\mathbf{v}, q)=\sum_{\mathrm{E} \in \Omega_{h}} b^{\mathrm{E}}(\mathbf{v}, \mathbf{w}) \quad \text { with } \quad b^{\mathrm{E}}(\mathbf{v}, q)=-\int_{\mathrm{E}} q \operatorname{div} \mathbf{v} d \mathbf{x} .
$$

We split $b_{h}(\cdot, \cdot)$ as the sum of the local bilinear forms $b_{h}^{\mathrm{E}}(\cdot, \cdot): \mathbf{V}_{k}^{h}(\mathrm{E}) \times \mathbb{P}_{\underline{k}}(\mathrm{E}) \rightarrow \mathbb{R}$ to define its virtual element approximation:

$$
b_{h}\left(\mathbf{v}_{h}, q\right)=\sum_{\mathrm{E} \in \Omega_{h}} b_{h}^{\mathrm{E}}\left(\mathbf{v}_{h}, q\right) \quad \text { where } \quad b_{h}^{\mathrm{E}}\left(\mathbf{v}_{h}, q\right)=-\int_{\mathrm{E}} q \Pi_{\underline{k}}^{0, \mathrm{E}} \operatorname{div} \mathbf{v}_{h} d \mathbf{x} .
$$

It holds that $b_{h}^{\mathrm{E}}\left(\mathbf{v}_{h}, q\right)=b^{\mathrm{E}}\left(\mathbf{v}_{h}, q\right)$ for every vector-valued field $\mathbf{v}_{h} \in \mathbf{V}_{k}^{h}(\mathrm{E})$ and polynomial scalar function $q \in$ $\mathbb{P}_{\underline{k}}(\mathrm{E})$, where we recall that $b^{\mathrm{E}}(\cdot, \cdot)$ is defined in (31). This property is a straightforward consequence of the definition of the orthogonal projection operator $\Pi_{\underline{k}}^{0, \mathrm{E}}$. If we add this relation over all the mesh elements, we find that

$$
b_{h}\left(\mathbf{v}_{h}, q\right)=b\left(\mathbf{v}_{h}, q\right) \quad \forall \mathbf{v}_{h} \in \mathbf{V}_{k}^{h}, q \in \mathbb{P}_{\underline{k}}\left(\Omega_{h}\right)
$$

for all pairs $(k, \underline{k})$ with $\underline{k} \leq k-1$, which can be used to simplify the implementation of the method.

\subsection{The virtual element approximation of the right-hand side}

Let $\bar{k}$ be a nonnegative integer number. The right hand-side of equation (10) is written as

$$
\left\langle\mathbf{f}_{h}, \mathbf{v}_{h}\right\rangle=\sum_{\mathrm{E} \in \Omega_{h}} \int_{\mathrm{E}} \Pi_{\bar{k}}^{0, \mathrm{E}} \mathbf{f} \cdot \mathbf{v}_{h} d \mathbf{x}=\sum_{\mathrm{E} \in \Omega_{h}} \int_{\mathrm{E}} \mathbf{f} \cdot \Pi_{\bar{k}}^{0, \mathrm{E}} \mathbf{v}_{h} d \mathbf{x},
$$


where the vector-valued source term $\mathbf{f}$ is locally approximated by its orthogonal projection $\Pi_{\bar{k}}^{0, \mathrm{E}} \mathbf{f}$ onto the polynomial space $\mathbb{P}_{\bar{k}}(\mathrm{E})$ for all polygonal elements $\mathrm{E}$. Note that we use the definition of the orthogonal projector $\Pi_{\bar{k}}^{0, \mathrm{E}}$ in the second equality above.

Two choices of $\bar{k}$ are possible:

- $\bar{k}=\max (0, k-2)$ if we consider the virtual element space in (13);

- $\bar{k}=k$ (or $k-1$ ): if we consider the enhanced virtual element space in (14).

The first choice was proposed for $k \geq 2$ in the original paper [8]. It is worth mentioning that for $k=1$ the projector $\Pi_{0}^{0, \mathrm{E}}$ is substituted by the arithmetic average of the elemental vertex values for each velocity vector component. This choice allows us to obtain the correct convergence rate for the approximation of the velocity field in the energy norm. The second choice was proposed in Ref. [2] and allows us to obtain the correct convergence rate for the approximation of the velocity field in the $L^{2}$-norm.

We recall the error estimates pertaining these two possible approximations of the right-hand side, which follow on noting that $\left(1-\Pi_{\bar{k}}^{0, \mathrm{E}}\right) \mathbf{f}_{h}$ is orthogonal to $\Pi_{0}^{0, \mathrm{E}} \mathbf{v}_{h}$ in the $L^{2}$-inner product. Assuming that $\mathbf{f} \in\left[H^{\bar{k}+1}(\Omega)\right]^{2}$ with $\bar{k} \geq 0$ we find that $[8]$ :

$$
\begin{aligned}
\left|\left\langle\mathbf{f}_{h}, \mathbf{v}_{h}\right\rangle-\left(\mathbf{f}, \mathbf{v}_{h}\right)\right| & =\left|\sum_{\mathrm{E} \in \Omega_{h}} \int_{\mathrm{E}}\left(\Pi_{\bar{k}}^{0, \mathrm{E}} \mathbf{f}-\mathbf{f}\right) \mathbf{v}_{h} d \mathbf{x}\right| \\
& \leq \sum_{\mathrm{E} \in \Omega_{h}}\left|\int_{\mathrm{E}}\left(\Pi_{\bar{k}}^{0, \mathrm{E}} \mathbf{f}-\mathbf{f}\right)\left(\mathbf{v}_{h}-\Pi_{0}^{0, \mathrm{E}} \mathbf{v}_{h}\right) d \mathbf{x}\right| \\
& \leq \sum_{\mathrm{E} \in \Omega_{h}}\left\|\Pi_{\bar{k}}^{0, \mathrm{E}} \mathbf{f}-\mathbf{f}\right\|_{0, \mathrm{E}}\left\|\mathbf{v}_{h}-\Pi_{0}^{0, \mathrm{E}} \mathbf{v}_{h}\right\|_{0, \mathrm{E}} \\
& \leq C h^{\bar{k}+2}\|\mathbf{f}\|_{\bar{k}+1, \mathrm{E}}\left|\mathbf{v}_{h}\right|_{1, \mathrm{E}},
\end{aligned}
$$

for some constant $C>0$ independent of $h$. For $\bar{k}=0$ and assuming $\mathbf{f} \in\left[L^{2}(\Omega)\right]^{2}$, we find that

$$
\begin{aligned}
\left|\left\langle\mathbf{f}_{h}, \mathbf{v}_{h}\right\rangle-\left(\mathbf{f}, \mathbf{v}_{h}\right)\right| & =\left|\sum_{\mathrm{E} \in \Omega_{h}} \int_{\mathrm{E}}\left(\Pi_{0}^{0, \mathrm{E}} \mathbf{f}-\mathbf{f}\right) \mathbf{v}_{h} d \mathbf{x}\right| \\
& \leq \sum_{\mathrm{E} \in \Omega_{h}}\left|\int_{\mathrm{E}}\left(\Pi_{0}^{0, \mathrm{E}} \mathbf{f}-\mathbf{f}\right)\left(\mathbf{v}_{h}-\Pi_{0}^{0, \mathrm{E}} \mathbf{v}_{h}\right) d \mathbf{x}\right| \\
& \leq \sum_{\mathrm{E} \in \Omega_{h}}\left\|\Pi_{0}^{0, \mathrm{E}} \mathbf{f}-\mathbf{f}\right\|_{0, \mathrm{E}}\left\|\mathbf{v}_{h}-\Pi_{0}^{0, \mathrm{E}} \mathbf{v}_{h}\right\|_{0, \mathrm{E}} \\
& \leq C h\|\mathbf{f}\|_{0, \mathrm{E}}\left|\mathbf{v}_{h}\right|_{1, \mathrm{E}},
\end{aligned}
$$

for some constant $C>0$ independent of $h$.

\section{Convergence theory in the energy norm}

In this section, we present an abstract convergence result for the VEM based on the pair of finite dimensional functional spaces $\left(\mathbf{V}_{k}^{h}, Q_{k-1}^{h}\right)$, see Theorem 4.8. This result allows us to derive an estimate for the approximation error on the velocity and the pressure, see Corollary 4.10. To carry out the convergence analysis in Section 4.3, we need a few preliminary results, which are reported in Section 4.1 , and to prove that the bilinear form $b_{h}(\cdot, \cdot)$ is inf-sup stable on $\mathbf{V}_{k}^{h} \times Q_{k-1}^{h}$ on the mesh families satisfiyng Assumptions (M1)-(M2), see Section 4.2.

\subsection{Preliminary results}

In the analysis of the next sections, we make use of the interpolants of the (smooth enough) vector-valued field $\mathbf{v}$ and scalar function $q$, respectively. The interpolants are the vector-valued field $\mathbf{v}_{I}$ in $\mathbf{V}_{k}^{h}$ and the scalar polynomial $q_{I}$ 
in $Q_{k-1}^{h}$ that have the same degrees of freedom of $\mathbf{v}$ and $q$. In this section, we report three technical lemmas regarding $\mathbf{v}_{I}$ and $q_{I}$. The first two lemmas state basic results for the approximation of a vector-valued field by its virtual element interpolant and a scalar function by its polynomial interpolant onto the subspace of polynomials, and are presented without a proof. The third lemma presents an identity that is used in the proof of Theorem 4.8.

Lemma 4.1 (Projection error $[8,38])$ Under Assumptions (M1)-(M2), for every vector-valued field $\mathbf{v} \in\left[H^{s+1}(E)\right]^{2}$ and scalar function $q \in H^{s}(E)$ with $1 \leq s \leq \ell$, there exists a vector polynomial $\mathbf{v}_{\pi} \in\left[\mathbb{P}_{\ell}(E)\right]^{2}$ and a scalar polynomial $q_{\pi} \in \mathbb{P}_{\ell-1}(E)$ such that

$$
\begin{aligned}
& \left\|\mathbf{v}-\mathbf{v}_{\pi}\right\|_{0, E}+h_{E}\left|\mathbf{v}-\mathbf{v}_{\pi}\right|_{1, E} \leq C h_{E}^{s+1}|\mathbf{v}|_{s+1, E}, \\
& \| q-q_{\pi}||_{0, E}+h_{E}\left|q-q_{\pi}\right|_{1, E} \leq C h_{E}^{s}|q|_{s, E}
\end{aligned}
$$

for some positive constant $C$ that is independent of $h_{E}$ but may depend on the polynomial degree $\ell$ and the mesh regularity constant $\varrho$.

Lemma 4.2 (Interpolation error $[8,38])$ Under Assumptions (M1)-(M2), for every vector-valued field $\mathbf{v} \in\left[H^{s+1}(E)\right]^{2}$ and scalar function $q \in H^{s}(E)$ with $1 \leq s \leq \ell$, there exists a vector valued-field $\mathbf{v}_{I} \in \mathbf{V}_{k}^{h}(E)$ and a scalar field $q_{I} \in \mathbb{P}_{\ell-1}(E)$ such that

$$
\begin{array}{r}
\| \mathbf{v}-\mathbf{v}_{I}||_{0, E}+h_{E}\left|\mathbf{v}-\mathbf{v}_{I}\right|_{1, E} \leq C h_{E}^{s+1}|\mathbf{v}|_{s+1, E} \\
\quad|| q-q_{I} \|_{0, E}+h_{E}\left|q-q_{I}\right|_{1, E} \leq C h_{E}^{s}|q|_{s, E}
\end{array}
$$

for some positive constant $C$ that is independent of $h_{E}$ but may depend on the polynomial degree $\ell$ and the mesh regularity constant $\varrho$.

Lemma 4.3 (Orthogonality between $\operatorname{div}\left(\mathbf{u}_{h}-\mathbf{u}_{I}\right)$ and $\left.p_{h}-p_{I}\right)$ Let $\mathbf{u} \in\left[H_{0}^{1}(\Omega)\right]^{2}$ be the exact solution of the variational formulation of the Stokes problem given in (7)-(8). Let $\left(\mathbf{u}_{h}, p_{h}\right) \in \mathbf{V}_{k}^{h} \times Q_{k-1}^{h}$ be the solution of the virtual element approximation (10)-(11). Then, it holds that

$$
b\left(\mathbf{u}_{h}-\mathbf{u}_{I}, p_{h}-p_{I}\right)=0 .
$$

Proof. First, note that the composed operator $\Pi_{k-1}^{0, \mathrm{E}} \operatorname{div}(\cdot)$ only depends on the degrees of freedom of its argument. These degrees of freedom are the same for $\mathbf{u}$ and its virtual element interpolation $\mathbf{u}_{I}$, so that it must hold that $\Pi_{k-1}^{0, \mathrm{E}} \operatorname{div} \mathbf{u}=\Pi_{k-1}^{0, \mathrm{E}} \operatorname{div} \mathbf{u}_{I}$. Using this property and the definition of the orthogonal projection $\Pi_{k-1}^{0, \mathrm{E}}$ yield

$$
\begin{aligned}
b(\mathbf{u}, q) & =\sum_{\mathrm{E}} \int_{\mathrm{E}} q \operatorname{div} \mathbf{u} d \mathbf{x}=\sum_{\mathrm{E}} \int_{\mathrm{E}} q \Pi_{k-1}^{0, \mathrm{E}} \operatorname{div} \mathbf{u} d \mathbf{x}=\sum_{\mathrm{E}} \int_{\mathrm{E}} q \Pi_{k-1}^{0, \mathrm{E}} \operatorname{div} \mathbf{u}_{I} d \mathbf{x} \\
& =\sum_{\mathrm{E}} \int_{\mathrm{E}} q \operatorname{div} \mathbf{u}_{I} d \mathbf{x}=b\left(\mathbf{u}_{I}, q\right)
\end{aligned}
$$

for every $q \in \mathbb{P}_{k-1}(\mathrm{E})$. Eventually, we note that $b\left(\mathbf{u}_{I}, q\right)=b(\mathbf{u}, q)=0$ from Eq. (8) and $b\left(\mathbf{u}_{h}, q\right)=b_{h}\left(\mathbf{u}_{h}, q\right)=0$ from Eqs. (33) and (11). So, it holds that $b\left(\mathbf{u}_{h}-\mathbf{u}_{I}, q\right)=0$, and relation (A.5) immediately follows by setting $q=p_{h}-p_{I}$.

\subsection{Discrete Inf-sup stability}

The main result of this section is the discrete inf-sup stability property that is stated by the following theorem. Theorem 4.4 Let $\mathbf{V}_{k}^{h}$ and $Q_{k-1}^{h}$ the virtual element space and the piecewise polynomial space respectively defined on meshes satisfying Assumptions (M1) - (M2) for $k \geq 2$ in (12) and (16), where $\mathbf{V}_{k}^{h}(E)$ can be either the regular local VEM space defined by (13) or the enhanced VEM space defined by (14). Then, there exists a real positive constant $\beta$ (independent of $h$ ) such that

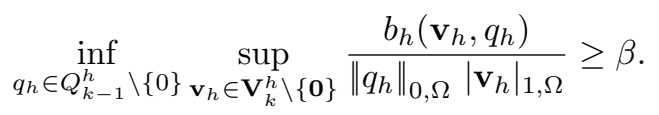


Proof. To prove this result, we exploit the inf-sup inequality of [23, Proposition 4.3] and the result of [53, Lemma 2 and Proposition 1]. We report these results below for completeness of exposition, with some (very minor) notational modifications to adapt them to the present context. The proof and a detailed discussion can be found in the cited references.

Proposition 4.5 (Proposition 4.3 from Reference [23]) Consider the virtual element space of vector-valued functions:

$$
\widetilde{\mathbf{V}}_{k}^{h}=\left\{\widetilde{\mathbf{v}}_{h} \in\left[H_{0}^{1}(\Omega)\right]^{2} \text { such that } \widetilde{\mathbf{v}}_{h \mid E} \in \widetilde{\mathbf{V}}_{k}^{h}(E) \forall E \in \Omega_{h}\right\}
$$

where the local vector space on every element $E$ is defined as

$$
\begin{aligned}
\widetilde{\mathbf{V}}_{k}^{h}(E)=\left\{\widetilde{\mathbf{v}}_{h} \in\left[H^{1}(E)\right]^{2}:\right. & : \widetilde{\mathbf{v}}_{h \mid \partial E} \in\left[C^{0}(\partial E)\right]^{2}, \widetilde{\mathbf{v}}_{h \mid e} \in\left[\mathbb{P}_{k}(e)\right]^{2} \forall e \in \partial E, \\
& -\Delta \widetilde{\mathbf{v}}_{h}-\nabla s \in \mathcal{G}_{k-2}(E)^{\perp} \quad \text { for some } s \in L^{2}(E), \\
& \left.\operatorname{div} \widetilde{\mathbf{v}}_{h} \in \mathbb{P}_{k-1}(E)\right\},
\end{aligned}
$$

and $\mathcal{G}_{k-2}(E)^{\perp}$ is the $L^{2}$-orthogonal complement to $\mathcal{G}_{k-2}(E)=\nabla \mathbb{P}_{k-1}(E)$. Then, there exists a real positive constant $\widetilde{\beta}$ (independent of $h$ ) such that

$$
\inf _{q_{h} \in Q_{k-1}^{h} \backslash\{0\}} \sup _{\widetilde{\mathbf{v}}_{h} \in \widetilde{\mathbf{V}}_{k}^{h} \backslash\{\mathbf{0}\}} \frac{b_{h}\left(\widetilde{\mathbf{v}}_{h}, q_{h}\right)}{\left\|q_{h}\right\|_{0, \Omega}\left|\widetilde{\mathbf{v}}_{h}\right|_{1, \Omega}} \geq \widetilde{\beta} .
$$

The virtual element space $\widetilde{\mathbf{V}}_{k}^{h}$ that is considered in Proposition 4.5 is rather different than the one that is considered in our work. However, it is proved in [53, Lemma 2] that we can establish a bijective correspondance mapping the degrees of freedom characterizing the "Stokes-like" virtual element fields in $\widetilde{\mathbf{V}}_{k}^{h}$ (see also [23] for their definition) and those characterizing the "Poisson-like" virtual element fields that are discussed in Section 3.3, cf. (12). Since the degrees of freedom are the same for both the regular and the enhanced space definitions (13) and (14), the argument that we present below holds for both situations. Let $\mathrm{T}_{S \mid P}^{\mathrm{E}}: \widetilde{\mathbf{V}}_{k}^{h}(\mathrm{E}) \rightarrow \mathrm{V}_{k}^{h}(\mathrm{E})$ denote this mapping. We report the result of [53, Proposition 1] as follows.

Proposition 4.6 (Proposition 1 from [53]) For all $\widetilde{\mathbf{v}}_{h} \in \widetilde{\mathbf{V}}_{k}^{h}$ we have

$$
b\left(T_{S \mid P}^{E} \widetilde{\mathbf{v}}_{h}, q\right)=b\left(\widetilde{\mathbf{v}}_{h}, q\right) \quad \forall q \in Q_{k}^{h}
$$

and

$$
\Pi_{k}^{\nabla}\left(T_{S \mid P}^{E} \widetilde{\mathbf{v}}_{h}\right)=\Pi_{k}^{\nabla}\left(\widetilde{\mathbf{v}}_{h}\right), \quad \Pi_{k-2}^{0}\left(T_{S \mid P}^{E} \widetilde{\mathbf{v}}_{h}\right)=\Pi_{k-2}^{0}\left(\widetilde{\mathbf{v}}_{h}\right) .
$$

The virtual element spaces $\mathbf{V}_{k}^{h}$ (this paper) and $\widetilde{\mathbf{V}}_{k}^{h}$ (Reference [23]) are substantially different although they both have the same vector polynomial subspace. Moreover, the virtual element vector-valued fields in them are uniquely identified by rather different sets of degrees of freedom (we refer again to [23] for their definition in $\widetilde{\mathbf{V}}_{k}^{h}$ ). Nonetheless, Proposition 4.6 above implies that two functions $\mathbf{v}_{h} \in \mathbf{V}_{k}^{h}$ and $\widetilde{\mathbf{v}}_{h} \in \widetilde{\mathbf{V}}_{k}^{h}$ have the same polynomial projections whenever they are in the bijection correspondance through $\mathrm{T}_{S \mid P}^{\mathrm{E}}$. As a consequence, the discrete bilinear forms $a_{h}(\cdot, \cdot)$ and $b_{h}(\cdot, \cdot)$ and the right-hand side functional $\left\langle\mathbf{f}_{h}, \cdot\right\rangle$, which only depend on such projection operators, must evaluate the same on corresponding functions. So, starting from Proposition 4.6, i.e., Reference [53, Proposition 1], we find that

$$
\begin{array}{rlrl}
b\left(\mathbf{v}_{h}, q_{h}\right) & =b\left(\widetilde{\mathbf{v}}_{h}, q_{h}\right) & & \\
& \geq \widetilde{\beta}\left|\widetilde{\mathbf{v}}_{h}\right|_{1, \Omega}\left\|q_{h}\right\|_{0, \Omega} & & \\
& \geq \widetilde{\beta}\left(\widetilde{\alpha}^{*}\right)^{-1} a_{h}\left(\widetilde{\mathbf{v}}_{h}, \widetilde{\mathbf{v}}_{h}\right)^{\frac{1}{2}}\left\|q_{h}\right\|_{0, \Omega} & {[\text { use again [23, Proposition 4.3] }]} \\
& =\widetilde{\beta}\left(\widetilde{\alpha}^{*}\right)^{-1} a_{h}\left(\mathbf{v}_{h}, \mathbf{v}_{h}\right)^{\frac{1}{2}}\left\|q_{h}\right\|_{0, \Omega} & {[\text { use (26)] }} \\
& \geq \widetilde{\beta}\left(\widetilde{\alpha}^{*}\right)^{-1} \alpha_{*}\left|\mathbf{v}_{h}\right|_{1, \Omega}\left\|q_{h}\right\|_{0, \Omega}, &
\end{array}
$$

which is the assertion of the theorem after setting $\beta=\widetilde{\beta}\left(\widetilde{\alpha}^{*}\right)^{-1} \alpha_{*}$ and noting that this real constant is independent of $h$. 
Remark 4.7 An alternative proof of the inf-sup inequality is also possible by adapting the argument used to prove such inequality in the virtual element method for linear elasticity that is found in [9].

\subsection{Convergence in the energy norm}

Theorem 4.8 (Abstract convergence result) Let $(\mathbf{u}, p) \in\left[H^{1+s}(\Omega) \cap H_{0}^{1}(\Omega)\right]^{2} \times L_{0}^{2}(\Omega), s \geq 1$, denote the solution of the variational formulation of the Stokes problem given in (7)-(8). Let $\left(\mathbf{u}_{h}, p_{h}\right) \in \mathbf{V}_{k}^{h} \times Q_{k-1}^{h}$ denote the solution of the virtual element variational formulation (10)-(11) for any polynomial degree $k \geq 2$ on the mesh family $\left\{\Omega_{h}\right\}_{h}$ satisfying the mesh regularity assumptions $(\mathbf{M 1})-(\mathbf{M 2})$. Then, the solution pair $\left(\mathbf{u}_{h}, p_{h}\right) \in \mathbf{V}_{k}^{h} \times Q_{k-1}^{h}$ exists and is unique, and for every interpolation approximation $\mathbf{u}_{I} \in \mathbf{V}_{k}^{h}$ and piecewise polynomial approximation $\mathbf{u}_{\pi} \in$ $\left[\mathbb{P}_{k}\left(\Omega_{h}\right)\right]^{2}$, the following abstract estimate holds:

$$
\begin{aligned}
\left|\mathbf{u}-\mathbf{u}_{h}\right|_{1, \Omega}+|| p-\left.p_{h}\right|_{0, \Omega} \leq C & || \mathbf{u}-\left.\mathbf{u}_{I}\right|_{1, \Omega}+\left|\mathbf{u}-\mathbf{u}_{\pi}\right|_{1, h}+|| p-\left.p_{I}\right|_{0, \Omega} \\
& \left.+\sup _{\mathbf{v}_{h} \in \mathbf{V}_{k}^{h} \backslash\{\mathbf{0}\}} \frac{\left|\left\langle\mathbf{f}_{h}, \mathbf{v}_{h}\right\rangle-\left(\mathbf{f}, \mathbf{v}_{h}\right)\right|}{\left|\mathbf{v}_{h}\right|_{1, \Omega}}\right)
\end{aligned}
$$

for some real, strictly positive constant $C$ independent of $h$.

Proof. First, we note that the stability property (26) and the Cauchy-Schwarz inequality imply that

$$
\begin{aligned}
C\left|\mathbf{v}_{h}\right|_{1, \Omega}^{2} \leq a_{h}\left(\mathbf{v}_{h}, \mathbf{v}_{h}\right) & & \forall \mathbf{v}_{h} \in \mathbf{V}_{k}^{h}, \\
\left|a_{h}\left(\mathbf{v}_{h}, \mathbf{w}_{h}\right)\right| \leq C\left|\mathbf{v}_{h}\right|_{1, \Omega}\left|\mathbf{w}_{h}\right|_{1, \Omega} & & \forall \mathbf{v}_{h}, \mathbf{w}_{h} \in \mathbf{V}_{k}^{h},
\end{aligned}
$$

for some real, strictly positive constant $C$. The bilinear form $b_{h}(\cdot, \cdot)$ is bounded since an application of the CauchySchwarz inequality yields that

$$
\left|b_{h}\left(\mathbf{v}_{h}, q\right)\right| \leq C\left|\mathbf{v}_{h}\right|_{1, \Omega}|| q \|_{0, \Omega} \quad \forall \mathbf{v}_{h} \in \mathbf{V}_{k}^{h}, q \in Q_{k-1}^{h} .
$$

According to the theory of saddle-point problems, see [37], the well-posedness of the VEM follows from the coercivity and boundedness of the bilinear form $a_{h}(\cdot, \cdot)$ and the discrete inf-sup inequality (42) and boundedness of the bilinear form $b_{h}(\cdot, \cdot)$.

To prove inequality (48), we introduce the virtual element field $\boldsymbol{\delta}_{h}=\mathbf{u}_{h}-\mathbf{u}_{I} \in \mathbf{V}_{k}^{h}$. The coercivity of $a_{h}(\cdot, \cdot)$, cf. Eq. (49), implies that:

$$
C\left|\boldsymbol{\delta}_{h}\right|_{1, \Omega}^{2} \leq a_{h}\left(\boldsymbol{\delta}_{h}, \boldsymbol{\delta}_{h}\right)=a_{h}\left(\mathbf{u}_{h}, \boldsymbol{\delta}_{h}\right)-a_{h}\left(\mathbf{u}_{I}, \boldsymbol{\delta}_{h}\right) .
$$

Then, we use (10) and (24) and add and substract $\mathbf{u}_{\pi}$, we use (33) and (A.5), which implies that $b_{h}\left(\boldsymbol{\delta}_{h}, p_{h}\right)=$ $b\left(\boldsymbol{\delta}_{h}, p_{h}\right)=b\left(\boldsymbol{\delta}_{h}, p_{I}\right)$, we use the consistency relation (27) for every local bilinear form $a_{h}^{\mathrm{E}}(\cdot, \cdot)$, and we finally add and substract $\mathbf{u}$ to the last term to obtain:

$$
\begin{aligned}
C\left|\boldsymbol{\delta}_{h}\right|_{1, \Omega}^{2} \leq & \left\langle\mathbf{f}_{h}, \boldsymbol{\delta}_{h}\right\rangle-b_{h}\left(\boldsymbol{\delta}_{h}, p_{h}\right)-\sum_{\mathrm{E} \in \Omega_{h}}\left(a_{h}^{\mathrm{E}}\left(\mathbf{u}_{I}-\mathbf{u}_{\pi}, \boldsymbol{\delta}_{h}\right)+a_{h}^{\mathrm{E}}\left(\mathbf{u}_{\pi}, \boldsymbol{\delta}_{h}\right)\right) \\
= & \left\langle\mathbf{f}_{h}, \boldsymbol{\delta}_{h}\right\rangle-b\left(\boldsymbol{\delta}_{h}, p_{I}\right)-\sum_{\mathrm{E} \in \Omega_{h}}\left(a_{h}^{\mathrm{E}}\left(\mathbf{u}_{I}-\mathbf{u}_{\pi}, \boldsymbol{\delta}_{h}\right)+a^{\mathrm{E}}\left(\mathbf{u}_{\pi}, \boldsymbol{\delta}_{h}\right)\right) \\
= & \left\langle\mathbf{f}_{h}, \boldsymbol{\delta}_{h}\right\rangle-b\left(\boldsymbol{\delta}_{h}, p_{I}\right)-\sum_{\mathrm{E} \in \Omega_{h}} a_{h}^{\mathrm{E}}\left(\mathbf{u}_{I}-\mathbf{u}_{\pi}, \boldsymbol{\delta}_{h}\right) \\
& -\sum_{\mathrm{E} \in \Omega_{h}}\left(a^{\mathrm{E}}\left(\mathbf{u}_{\pi}-\mathbf{u}, \boldsymbol{\delta}_{h}\right)+a^{\mathrm{E}}\left(\mathbf{u}, \boldsymbol{\delta}_{h}\right)\right) .
\end{aligned}
$$

We use (23), (7), and rearrange the terms: 


$$
\begin{aligned}
& C\left|\boldsymbol{\delta}_{h}\right|_{1, \Omega}^{2} \leq\left\langle\mathbf{f}_{h}, \boldsymbol{\delta}_{h}\right\rangle-b\left(\boldsymbol{\delta}_{h}, p_{I}\right)-\sum_{\mathrm{E} \in \Omega_{h}} a_{h}^{\mathrm{E}}\left(\mathbf{u}_{I}-\mathbf{u}_{\pi}, \boldsymbol{\delta}_{h}\right)-\sum_{\mathrm{E} \in \Omega_{h}} a^{\mathrm{E}}\left(\mathbf{u}_{\pi}-\mathbf{u}, \boldsymbol{\delta}_{h}\right) \\
&-a\left(\mathbf{u}, \boldsymbol{\delta}_{h}\right) \\
&=\left\langle\mathbf{f}_{h}, \boldsymbol{\delta}_{h}\right\rangle-b\left(\boldsymbol{\delta}_{h}, p_{I}\right)-\sum_{\mathrm{E} \in \Omega_{h}} a_{h}^{\mathrm{E}}\left(\mathbf{u}_{I}-\mathbf{u}_{\pi}, \boldsymbol{\delta}_{h}\right)-\sum_{\mathrm{E} \in \Omega_{h}} a^{\mathrm{E}}\left(\mathbf{u}_{\pi}-\mathbf{u}, \boldsymbol{\delta}_{h}\right) \\
&-\left(\left(\mathbf{f}, \boldsymbol{\delta}_{h}\right)-b\left(\boldsymbol{\delta}_{h}, p\right)\right) \\
&= {\left[\left\langle\mathbf{f}_{h}, \boldsymbol{\delta}_{h}\right\rangle-\left(\mathbf{f}, \boldsymbol{\delta}_{h}\right)\right]+\left[b\left(\boldsymbol{\delta}_{h}, p\right)-b\left(\boldsymbol{\delta}_{h}, p_{I}\right)\right] } \\
&+\left[-\sum_{\mathrm{E} \in \Omega_{h}} a_{h}^{\mathrm{E}}\left(\mathbf{u}_{I}-\mathbf{u}_{\pi}, \boldsymbol{\delta}_{h}\right)-\sum_{\mathrm{E} \in \Omega_{h}} a^{\mathrm{E}}\left(\mathbf{u}_{\pi}-\mathbf{u}, \boldsymbol{\delta}_{h}\right)\right] \\
&= {[(\mathbf{R} \mathbf{1})]+[(\mathbf{R 2})]+[(\mathbf{R 3})] . }
\end{aligned}
$$

At this point, we continue estimating separately the three terms $(\mathbf{R 1}),(\mathbf{R} 2)$, and $(\mathbf{R 3})$, which are identified in the last step of the above inequality chain by the square brackets.

Term $(\mathbf{R} 1)$ is bounded from above by multiplying and dividing by $\boldsymbol{\delta}_{h}$, and then taking the supremum

$$
\begin{aligned}
|(\mathbf{R} \mathbf{1})| & =\left|\left\langle\mathbf{f}_{h}, \boldsymbol{\delta}_{h}\right\rangle-\left(\mathbf{f}, \boldsymbol{\delta}_{h}\right)\right|=\frac{\left|\left\langle\mathbf{f}_{h}, \boldsymbol{\delta}_{h}\right\rangle-\left(\mathbf{f}, \boldsymbol{\delta}_{h}\right)\right|}{\left|\boldsymbol{\delta}_{h}\right|_{1, \Omega}}\left|\boldsymbol{\delta}_{h}\right|_{1, \Omega} \\
& \leq\left[\sup _{\mathbf{v}_{h} \in \mathbf{V}_{k}^{h} \backslash\{\mathbf{0}\}} \frac{\left|\left\langle\mathbf{f}_{h}, \mathbf{v}_{h}\right\rangle-\left(\mathbf{f}, \mathbf{v}_{h}\right)\right|}{\left|\mathbf{v}_{h}\right|_{1, \Omega}}\right]\left|\boldsymbol{\delta}_{h}\right|_{1, \Omega} .
\end{aligned}
$$

Term $(\mathbf{R} 2)$ is bounded from above by applying the Cauchy-Schwarz inequality to find that

$$
\begin{aligned}
|(\mathbf{R 2})| & =\left|b\left(\boldsymbol{\delta}_{h}, p-p_{I}\right)\right|=\left|\left(\operatorname{div} \boldsymbol{\delta}_{h}, p-p_{I}\right)\right| \leq\left\|\operatorname{div} \boldsymbol{\delta}_{h}\right\|_{0, \Omega}\left\|p-p_{I}\right\|_{0, \Omega} \\
& \leq\left|\boldsymbol{\delta}_{h}\right|_{1, \Omega}\left\|p-p_{I}\right\|_{0, \Omega} .
\end{aligned}
$$

Finally, term $(\mathbf{R 3})$ is bounded from above by using the local continuity of $a_{h}^{\mathrm{E}}(\cdot, \cdot)$, cf. (29), and $a^{\mathrm{E}}(\cdot, \cdot)$ to find that

$$
\begin{aligned}
|(\mathbf{R 3})| & =\left|\sum_{\mathrm{E} \in \Omega_{h}}\left(a_{h}^{\mathrm{E}}\left(\mathbf{u}_{I}-\mathbf{u}_{\pi}, \boldsymbol{\delta}_{h}\right)+a^{\mathrm{E}}\left(\mathbf{u}_{\pi}-\mathbf{u}, \boldsymbol{\delta}_{h}\right)\right)\right| \\
& \leq \sum_{\mathrm{E} \in \Omega_{h}}\left(\left|a_{h}^{\mathrm{E}}\left(\mathbf{u}_{I}-\mathbf{u}_{\pi}, \boldsymbol{\delta}_{h}\right)\right|+\left|a^{\mathrm{E}}\left(\mathbf{u}_{\pi}-\mathbf{u}, \boldsymbol{\delta}_{h}\right)\right|\right) \\
& \leq \sum_{\mathrm{E} \in \Omega_{h}}\left(\alpha^{*}\left|\mathbf{u}_{I}-\mathbf{u}_{\pi}\right|_{1, \mathrm{E}}+\left|\mathbf{u}-\mathbf{u}_{\pi}\right|_{1, \mathrm{E}}\right)\left|\boldsymbol{\delta}_{h}\right|_{1, \mathrm{E}} .
\end{aligned}
$$

Then, we add and subtract $\mathbf{u}$ in the first summation argument, and, in the last step, we introduce the broken seminorm $|\cdot|_{1, h}^{2}=\sum_{\mathrm{E} \in \Omega_{h}}|\cdot|_{1, \mathrm{E}}^{2}$ to find that

$$
\begin{aligned}
|(\mathbf{R} 3)| & \leq \sum_{\mathrm{E} \in \Omega_{h}}\left(\alpha^{*}\left|\mathbf{u}_{I}-\mathbf{u}\right|_{1, \mathrm{E}}+\left(1+\alpha^{*}\right)\left|\mathbf{u}-\mathbf{u}_{\pi}\right|_{1, \mathrm{E}}\right)\left|\boldsymbol{\delta}_{h}\right|_{1, \mathrm{E}} \\
& \leq\left(\alpha^{*}\left|\mathbf{u}-\mathbf{u}_{I}\right|_{1, \Omega}+\left(1+\alpha^{*}\right)\left|\mathbf{u}-\mathbf{u}_{\pi}\right|_{1, h}\right)\left|\boldsymbol{\delta}_{h}\right|_{1, \Omega} .
\end{aligned}
$$

We are left to derive a bound for the pressure term. To this end, we consider $\sigma_{h}=p_{h}-p_{I}$. Note that $p_{h}$ is the solution of (10)-(11) and the integral of $p_{I}$ on $\Omega$ is zero because $p_{I \mid \mathrm{E}}$ has the same degrees of freedom, i.e., polynomial moments, of $p_{\mid \mathrm{E}}$, so that 


$$
\int_{\Omega} p_{I} d \mathbf{x}=\sum_{\mathrm{E} \in \Omega_{h}} \int_{\mathrm{E}} p_{I} d \mathbf{x}=\sum_{\mathrm{E} \in \Omega_{h}} \int_{\mathrm{E}} p d \mathbf{x}=\int_{\Omega} p d \mathbf{x}=0
$$

Henceforth, the global interpolant $p_{I}$, which is built from the local interpolant that is such that $p_{I \mid \mathrm{E}}=\left(p_{\mid \mathrm{E}}\right)_{I} \in$ $Q_{k-1}^{h}(\mathrm{E})$, belongs to $Q_{k-1}^{h}$. We start the derivation of a bound for $\left\|\sigma_{h}\right\|_{0, \Omega}$ from the inf-sup condition (42), which implies that for every $\sigma_{h} \in Q_{k-1}^{h}$, there exists a vector field $\mathbf{v}_{h} \in \mathbf{V}_{k}^{h}$ such that

$$
\left.\beta|| \sigma_{h}\right|_{0, \Omega}\left|\mathbf{v}_{h}\right|_{1, \Omega} \leq b_{h}\left(\mathbf{v}_{h}, \sigma_{h}\right) .
$$

Then, we split $\sigma_{h}=p_{h}-p_{I}$, we use equation (10) and add (7) and we find that

$$
\begin{aligned}
& b_{h}\left(\mathbf{v}_{h}, \sigma_{h}\right)=b_{h}\left(\mathbf{v}_{h}, p_{h}\right)-b_{h}\left(\mathbf{v}_{h}, p_{I}\right)=-a_{h}\left(\mathbf{u}_{h}, \mathbf{v}_{h}\right)+\left\langle\mathbf{f}_{h}, \mathbf{v}_{h}\right\rangle-b_{h}\left(\mathbf{v}_{h}, p_{I}\right) \\
& \quad=-a_{h}\left(\mathbf{u}_{h}, \mathbf{v}_{h}\right)+\left\langle\mathbf{f}_{h}, \mathbf{v}_{h}\right\rangle-b_{h}\left(\mathbf{v}_{h}, p_{I}\right)+\left(a\left(\mathbf{u}, \mathbf{v}_{h}\right)+b\left(\mathbf{v}_{h}, p\right)-\left(\mathbf{f}, \mathbf{v}_{h}\right)\right) .
\end{aligned}
$$

We rearrange the terms, we use (23) and (24), we add and substract $\mathbf{u}_{\pi}$, we note again that $b_{h}\left(\mathbf{v}_{h}, p_{I}\right)=b\left(\mathbf{v}_{h}, p_{I}\right)$, use (27) with $\mathbf{q}=\mathbf{u}_{\pi}$ and we find that

$$
\begin{aligned}
& \left.\beta|| \sigma_{h}\right|_{0, \Omega}\left|\mathbf{v}_{h}\right|_{1, \Omega} \leq \sum_{\mathrm{E} \in \Omega_{h}}\left(a^{\mathrm{E}}\left(\mathbf{u}, \mathbf{v}_{h}\right)-a_{h}^{\mathrm{E}}\left(\mathbf{u}_{h}, \mathbf{v}_{h}\right)\right)+\left\langle\mathbf{f}_{h}, \mathbf{v}_{h}\right\rangle-\left(\mathbf{f}, \mathbf{v}_{h}\right) \\
& +b\left(\mathbf{v}_{h}, p\right)-b_{h}\left(\mathbf{v}_{h}, p_{I}\right) \\
& =\left[\left\langle\mathbf{f}_{h}, \mathbf{v}_{h}\right\rangle-\left(\mathbf{f}, \mathbf{v}_{h}\right)\right]+\left[b\left(\mathbf{v}_{h}, p\right)-b\left(\mathbf{v}_{h}, p_{I}\right)\right] \\
& +\sum_{\mathrm{E} \in \Omega_{h}}\left(a^{\mathrm{E}}\left(\mathbf{u}-\mathbf{u}_{\pi}, \mathbf{v}_{h}\right)-a_{h}^{\mathrm{E}}\left(\mathbf{u}_{h}-\mathbf{u}_{\pi}, \mathbf{v}_{h}\right)\right) \\
& =[(\mathbf{R 4})]+[(\mathbf{R 5})]+[(\mathbf{R 6})],
\end{aligned}
$$

where, again, terms $(\mathbf{R 4}),(\mathbf{R 5})$, and $(\mathbf{R 6})$ are identified by the square brackets. As for the term $\boldsymbol{\delta}_{h}$, we continue estimating each term separately.

Term $(\mathbf{R 4})$ is bounded from above as we bounded term $(\mathbf{R} \mathbf{1})$ with $\mathbf{v}_{h}$ instead of $\boldsymbol{\delta}_{h}$

$$
|(\mathbf{R 4})|=\left|\left\langle\mathbf{f}_{h}, \mathbf{v}_{h}\right\rangle-\left(\mathbf{f}, \mathbf{v}_{h}\right)\right| \leq\left[\sup _{\mathbf{v}_{h} \in \mathbf{V}_{k}^{h} \backslash\{\mathbf{0}\}} \frac{\left|\left\langle\mathbf{f}_{h}, \mathbf{v}_{h}\right\rangle-\left(\mathbf{f}, \mathbf{v}_{h}\right)\right|}{\left|\mathbf{v}_{h}\right|_{1, \Omega}}\right]\left|\mathbf{v}_{h}\right|_{1, \Omega} .
$$

Term $(\mathbf{R 5})$ is bounded from above as we bounded term $(\mathbf{R} 2)$ with $\mathbf{v}_{h}$ instead of $\boldsymbol{\delta}_{h}$

$$
|(\mathbf{R 5})| \leq\left|\mathbf{v}_{h}\right|_{1, \Omega}|| p_{I}-\left.p\right|_{0, \Omega} .
$$

Term $(\mathbf{R 6})$ is bounded from above as we bounded term $(\mathbf{R 3})$ with $\mathbf{v}_{h}$ instead of $\boldsymbol{\delta}_{h}$

$$
|(\mathbf{R 6})| \leq\left(\alpha^{*}\left|\mathbf{u}-\mathbf{u}_{I}\right|_{1, \Omega}+\left(1+\alpha^{*}\right)\left|\mathbf{u}-\mathbf{u}_{\pi}\right|_{1, h}\right)\left|\mathbf{v}_{h}\right|_{1, \Omega},
$$

Eventually, we obtain the energy estimate of inequality (48) by adding and substracting $\mathbf{u}_{I}$ and $p_{I}$ in the two terms of the left-hand side of (48) and using the triangle inequalities

$$
\begin{aligned}
\left|\mathbf{u}-\mathbf{u}_{h}\right|_{1, \Omega} & \leq\left|\mathbf{u}-\mathbf{u}_{I}\right|_{1, \Omega}+\left|\mathbf{u}_{I}-\mathbf{u}_{h}\right|_{1, \Omega}, \\
|| p-\left.p_{h}\right|_{0, \Omega} & \leq|| p-\left.p_{I}\right|_{0, \Omega}+\left|p_{I}-p_{h}\right|_{0, \Omega} .
\end{aligned}
$$

We estimate the first term in the right-hand side of the two inequalities above by using standard estimates of the interpolation errors provided by Lemma A.1 and the second term by substituting the bounds we previously derived for the terms $(\mathbf{R 1})-(\mathbf{R 6})$.

Remark 4.9 In the proof of Theorem 4.8, we need to deal with a pressure dependent term in the estimate of the velocity error, e.g., term (R2). A different approach permits the derivation of an estimate of the velocity error that does not require to cope with pressure terms, see, e.g., [23, Theorem 4.6]. 

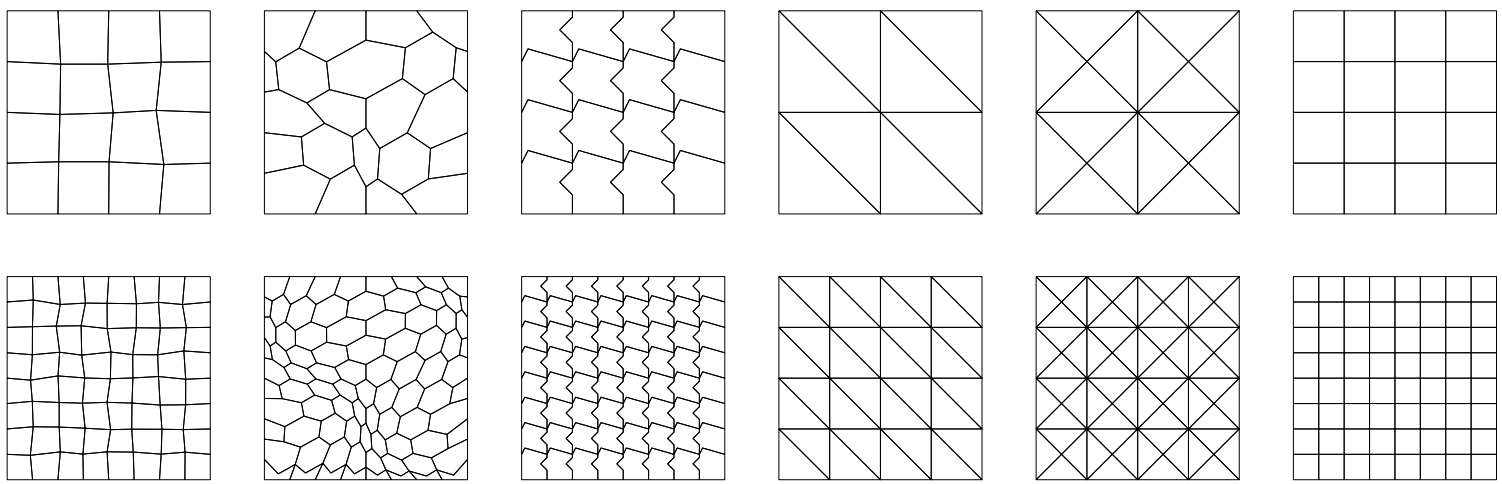

(a) (c) (d) (e)

Fig. 2. Base meshes (top row) and first refinement meshes (bottom row) of the six mesh families used in this section: $(a)$ randomly quadrilateral meshes; $(b)$ general polygonal meshes; $(c)$ concave element meshes; $(d)$ diagonal triangle meshes; $(e)$ criss-cross triangle meshes; $(f)$ square meshes.

An error estimate follows immediate from Theorem 4.8. We state it in the following corollary.

Corollary 4.10 (Error estimate) Let $\left(\mathbf{u}_{h}, p_{h}\right) \in \mathbf{V}_{k}^{h} \times Q_{k-1}^{h}, k \geq 2$, be the virtual element solution fields approximating $\mathbf{u} \in\left[H^{s+1}(\Omega) \cap H_{0}^{1}(\Omega)\right]^{2}$ and $p \in H^{s}(\Omega) \cap L_{0}^{2}(\Omega)$, and take $\mathbf{f} \in\left[H^{s}(\Omega)\right]^{2}$ for $1 \leq s \leq k$. Then, under the assumptions of Theorem 4.8, it holds that

$$
\left|\mathbf{u}-\mathbf{u}_{h}\right|_{1, \Omega}+\left\|p-p_{h}\right\|_{0, \Omega} \leq C h^{s}\left(\|\mathbf{u}\|_{s+1, \Omega}+\|p\|_{s, \Omega}+\|\mathbf{f}\|_{s, \Omega}\right)
$$

for some real, strictly positive constant $C$ independent of $h$.

Proof. Estimate (54) follows from a straightforward application of the results of Lemmas A.2 and A.1, and estimates (35)-(36) to the right-hand side of (48).

Remark 4.11 The abstract convergence result of Theorem 4.8 and the error estimate of Corollary 4.10 hold for $k \geq 2$. On the one hand, this limitation is a consequence of Theorem 4.4 , which states that the inf-sup stability holds for $k \geq 2$. On the other hand, a convergence result for $k=1$ does not hold in general since we know that this scheme can be unstable on some meshes, including triangular and quadrilateral meshes. An extension to $k=1$ is however possible by requiring the mesh elements to satisfy stronger conditions than Assumptions (M1) - (M2) and including edge bubble functions in the formulation of the method. An example of this approach is provided by the work of Reference [19].

\section{Numerical experiments}

We assess the convergence property of the virtual element formulation considered in this paper by numerically solving problem (7)-(8) on the computational domain $\Omega=[0,1] \times[0,1]$ partitioned by the six mesh families of Figure 2. Dirichlet boundary conditions and source terms are set accordingly to the manufactured solution $\mathbf{u}=$ $\left(u_{x}, u_{y}\right)^{T}$ and $p$ given by

$$
\begin{aligned}
& u_{x}(x, y)=\cos (2 \pi x) \sin (2 \pi y), \\
& u_{y}(x, y)=-\sin (2 \pi x) \cos (2 \pi y), \\
& p(x, y)=e^{x+y}-(e-1)^{2} .
\end{aligned}
$$

On any set of refined meshes, we measure the $H^{1}$ relative error for the velocity vector field by applying the formula

$$
\operatorname{error}_{H^{1}(\Omega)}(\mathbf{u})=\frac{\left|\mathbf{u}-\Pi_{k}^{0} \mathbf{u}_{h}\right|_{1, h}}{|\mathbf{u}|_{1, \Omega}} \approx \frac{\left|\mathbf{u}-\mathbf{u}_{h}\right|_{1, \Omega}}{|\mathbf{u}|_{1, \Omega}},
$$

and the $L^{2}$ relative error by applying the formula 


$$
\operatorname{error}_{L^{2}(\Omega)}(\mathbf{u})=\frac{\left\|\mathbf{u}-\Pi_{k}^{0} \mathbf{u}_{h}\right\|_{0, \Omega}}{\|\mathbf{u}\|_{0, \Omega}} \approx \frac{\left\|\mathbf{u}-\mathbf{u}_{h}\right\|_{0, \Omega}}{\|\mathbf{u}\|_{0, \Omega}} .
$$

For the pressure scalar field we measure the $L^{2}(\Omega)$ relative error by applying the formula

$$
\operatorname{error}_{L^{2}(\Omega)}(p)=\frac{\left\|p-p_{h}\right\|_{0, \Omega}}{\|p\|_{0, \Omega}}
$$

Discretization of the bilinear form $a_{h}(\cdot, \cdot)$ and $b_{h}(\cdot, \cdot)$ yields two matrices A and $\mathrm{B}$, respectively, while discretization of the right-hand side gives the vector $\mathbf{f}$.

To write the formal expression of such matrices we introduce the canonical shape functions $\Phi_{j}$ of the virtual element space $\mathbf{V}_{k}^{h}(\mathrm{E})$, and the basis functions $\mathbf{m}_{\alpha}$ of the local polynomial space $\left[\mathbb{P}_{\ell}(\mathrm{E})\right]^{2}$, wheer $\ell$ can be equal to $k-1$ or $k$. We can assume that space $\mathbb{P}_{\ell}(\mathrm{E})$ is the span of the finite set of scaled monomials of degree up to $\ell$, that are given by

$$
\mathcal{M}_{\ell}(\omega)=\left\{\left(\frac{\mathbf{x}-\mathbf{x}_{\omega}}{h_{\omega}}\right)^{\alpha} \text { with }|\alpha| \leq \ell\right\}
$$

where

- $\mathrm{x}_{\mathrm{E}}$ denotes the center of gravity of $\mathrm{E}$ and $h_{\mathrm{E}}$ its characteristic length, e.g., the cell diameter;

- $\alpha=\left(\alpha_{1}, \alpha_{2}\right)$ is the two-dimensional multi-index of nonnegative integers $\alpha_{i}$ with degree $|\alpha|=\alpha_{1}+\alpha_{2} \leq \ell$ and such that $\mathbf{x}^{\alpha}=x_{1}^{\alpha_{1}} x_{2}^{\alpha_{2}}$ for any $\mathbf{x} \in \mathbb{R}^{2}$ and $\partial^{|\alpha|} / \partial \mathbf{x}^{\alpha}=\partial^{|\alpha|} / \partial x_{1}^{\alpha_{1}} \partial x_{2}^{\alpha_{2}}$.

Alternatively, we can assume that space $\mathbb{P}_{\ell}(\mathrm{E})$ is the span of a set of orthogonalized polynomials built from the scaled monomials by applying the Gram-Schmidt process in all elements $\mathrm{E}$.

Noe, the entries of the global matrix A are given by assembling the local consistency and stability matrices,

$$
\mathrm{A}=\sum_{\mathrm{E}} \mathrm{Q}^{T}\left(\mathrm{~A}_{\mathrm{E}}^{C}+\mathrm{A}_{\mathrm{E}}^{S}\right) \mathrm{Q}
$$

where $\mathrm{Q}$ is the assembling matrix that remaps the local entries of the elemental matrices $\mathrm{A}_{\mathrm{E}}^{C}$ and $\mathrm{A}_{\mathrm{E}}^{S}$ into the global setting of matrix $A$, and

$$
\begin{aligned}
\left(\mathrm{A}_{\mathrm{E}}^{C}\right)_{i j} & =\int_{\mathrm{E}} \Pi_{k-1}^{0, \mathrm{E}} \nabla \Phi_{i}: \Pi_{k-1}^{0, \mathrm{E}} \nabla \Phi_{j} d \mathbf{x}, \\
\mathrm{A}_{\mathrm{E}}^{S} & =\left(I-\Pi^{\nabla}\right)^{T}\left(I-\Pi^{\nabla}\right)
\end{aligned}
$$

and $\Pi^{\nabla}$ is the matrix associated to the elliptic projector. Likewise, the global matrix B has entries given by assembling the local contributions of the integrals

$$
\left(\mathrm{B}_{\mathrm{E}}\right)_{\alpha j}=\int_{\mathrm{E}} \mathbf{m}_{\alpha} \Pi_{\underline{k}}^{0, \mathrm{E}} \operatorname{div} \Phi_{j} d \mathbf{x} .
$$

Denoting with $\mathbf{u}$ and $\mathbf{p}$ the vectors of velocity and pressure degrees of freedom, respectively, the problem to be solved reads as:

$$
\left[\begin{array}{cc}
\mathrm{A} & \mathrm{B}^{T} \\
\mathrm{~B} & 0
\end{array}\right]\left[\begin{array}{l}
\mathbf{u} \\
\mathbf{p}
\end{array}\right]=\left[\begin{array}{l}
\mathbf{f} \\
0
\end{array}\right]
$$

To solve this system, we eliminate the degrees of freedom corresponding to the Dirichlet conditions, and impose the additional condition that $\int_{\Omega} p d \mathbf{x}=0$.

\subsection{General convergence results}

We compare the approximation errors (55), (56), and (57) obtained by applying the numerical scheme to the mesh families $(a),(b)$, and $(c)$ and setting $\underline{k}=k-1$. Figure 3 shows the approximation errors when the right-hand side is discretized by using the projection operator $\Pi_{\bar{k}}^{0}$ with $\bar{k}=k$. Figure 4 shows the approximation errors when the right-hand side is approximated by using the projection operator $\Pi_{\bar{k}}^{0}$ with $\bar{k}=\max (0, k-2)$. When we set $\bar{k}=k$ we observe the optimal convergence rates in all the norms, so the error for the velocity in the energy norm and in the $L^{2}$ norm scales as $\mathcal{O}\left(h^{k}\right)$ and $\mathcal{O}\left(h^{k+1}\right)$, and the error of the pressure scales as $\mathcal{O}\left(h^{k}\right)$. When we set $\bar{k}=\max (0, k-2)$, 
we observe the optimal convergence rate for the velocity approximation for all $k \geq 1$ only in the energy norm. Instead, the velocity error in the $L^{2}$-norm looses an order of approximation when $k=2$. Moreover, an overall better approximation, i.e., smaller errors, are visible when we select $\bar{k}=k$, which corresponds to the enhanced virtual element space (14) Regarding the approximation of the zero-divergence constraint, we note that the divergence of the velocity is close to the machine precision for all meshes and $k$ here considered.

\subsection{The lowest-order case for $k=1$}

The case $k=1$ is critical since on triangular and square meshes this scheme coincides with the $P^{1}-P^{0}$ ScottVogelius method, which is indeed pathological. We experimentally investigate this issue by using the mesh families $(d),(e)$, and $(f)$. Following [71] and using orthonormal polynomials, a relationship between the inf-sup constant $\beta$ and the minimum non-zero eigenvalue of the matrix $B A^{-1} B^{T}$ is given by

$$
\beta=\sqrt{\lambda_{\min }\left(\mathrm{BA}^{-1} \mathrm{~B}^{T}\right)}
$$

where $\lambda_{\min }$ is the smallest non-zero eigenvalue, and matrices B and A are defined as in (58).

Note that we consider matrix $B$ after removing the rows and columns that correspond to the boundary degrees of freedom. If $l$ is the size of the kernel of matrix $\mathrm{B}$, the rank of $\mathrm{B}$ is equal to $m=n_{\bar{k}}-l$, and matrix $\mathrm{BA}^{-1} \mathrm{~B}^{T}$ has $l$ eigenvalues equal to zero. In Figure 5, we show the values of the inf-sup constant $\beta$ for $k=1$ versus the mesh size parameter $h$ of the diagonal, criss-cross and square meshes. It is evident from these plots that $\beta$ approaches zero, revealing that the inf-sup condition is not satisfied.

Moreover, the numerical approximations obtained with $k \geq 2$ and $\underline{k}=k-1$ give results as expected from the theory, as we can see from Figure 6.

\subsection{The case for $k=2$ and $\underline{k}=0$}

If we use the formulation with $k=2$ for the velocity virtual element space and $\underline{k}=0$ for the pressure, we observe that the velocity errors in the energy norm are very similar if the $\Pi_{k}^{0}$ or $\Pi_{k-2}^{0}$ projector is applied for the right-hand side and show a rate of convergence of order 2 . On the contrary, in $L^{2}$-norm, we note convergence of order between 2 and 3 when the projector $\Pi_{k}^{0}$ is applied, and convergence of of order 2 when the projector $\Pi_{k-2}^{0}$ is applied.

For the approximation of the scalar unknown, the rate is approaching 1 in both cases and we observe better results with the $\Pi_{k}^{0}$ projector. The results for the case $k=2, \underline{k}=0$ are shown in Figure 7.

\section{Conclusions}

We studied a conforming virtual element formulation that generalizes the Scott-Vogelius FEM for the numerical approximation of the Stokes problem to unstructured meshes and works at any order of accuracy. The components of the vector-valued unknown are approximated by using the conforming regular or enhanced virtual element approximation space that were originally introduced for the discretization of the Poisson equation. The scalar unknown is approximated by using discontinuous polynomials. The stiffness bilinear form is approximated by using the orthogonal polynomial projection of the gradients onto vector polynomials of degree $k-1$ and adding a suitable stabilization term. The zero divergence constraint is taken into account by projecting the divergence equation onto the space of polynomials of degree $k-1$. We presented a number of numerical experiments to demonstrate that this formulation is inf-sup stable and convergent with optimal convergence rates except for the lowest-order case (e.g., for the polynomial order $k=1$ ) on triangular meshes, which corresponds to the well-known $\mathbb{P}_{1}-\mathbb{P}_{0}$ unstable case of the Scott-Vogelius method, and squares meshes. Moreover, our numerical experiments show that the divergence constraint is satisfied at the machine precision level by the orthogonal polynomial projection of the divergence of the approximate velocity vector. 

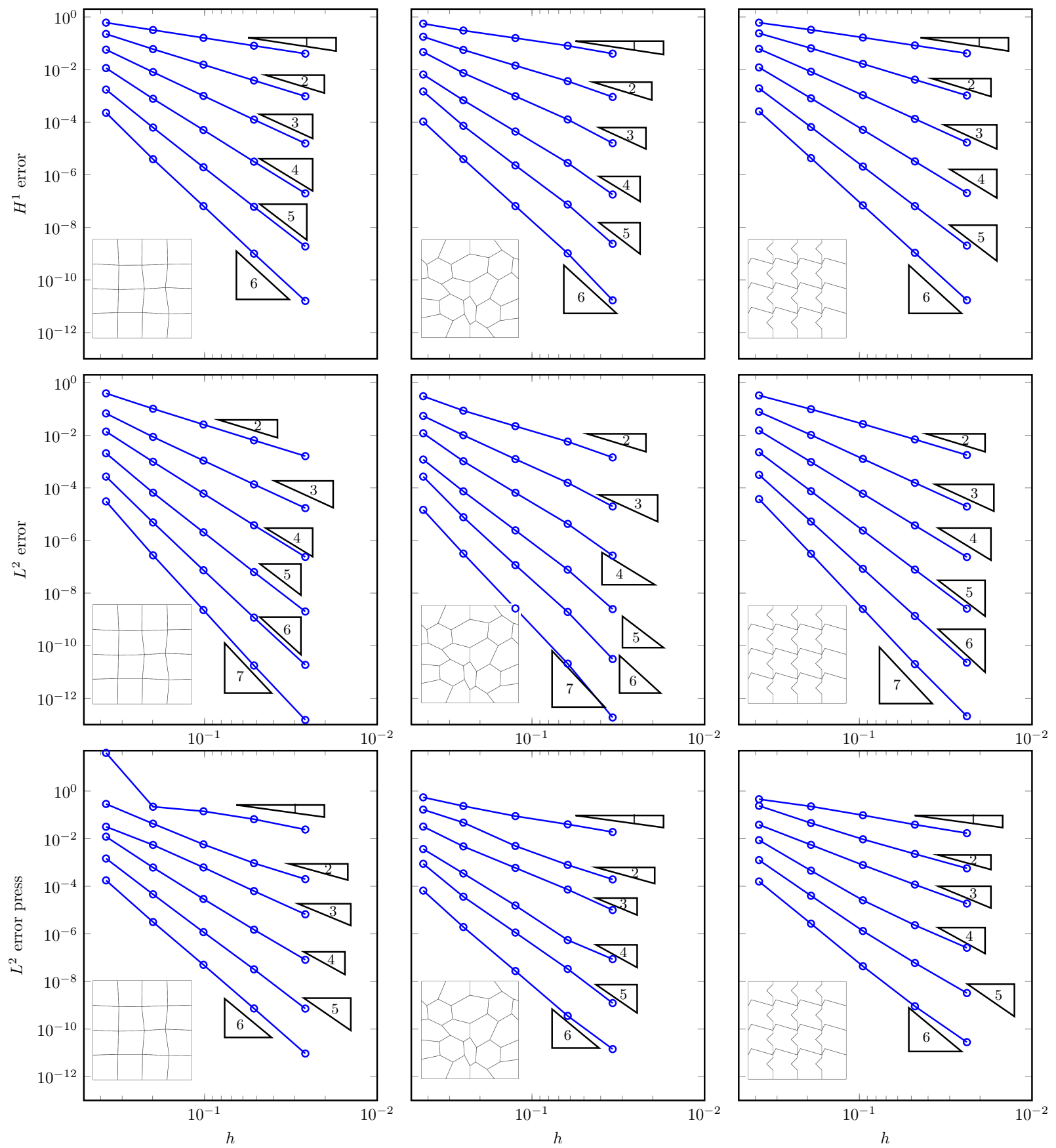

Fig. 3. Convergence curves versus the mesh size parameter $h$ for the velocity approximation measured using the energy norm (55) (top panels) and the $L^{2}$-norm (56) (mid panels), and for the pressure approximation measured using the $L^{2}$-norm (57) (bottom panels). Blue lines with circles represent the error curves for the Basic Formulation using the enhanced virtual element space (14). The right-hand side is approximated by using the projection operator $\Pi_{k}^{0}$. The mesh families used in each calculations are shown in the left corner of each panel. The expected convergence slopes and rates are shown by the triangles and corresponding numeric labels. 

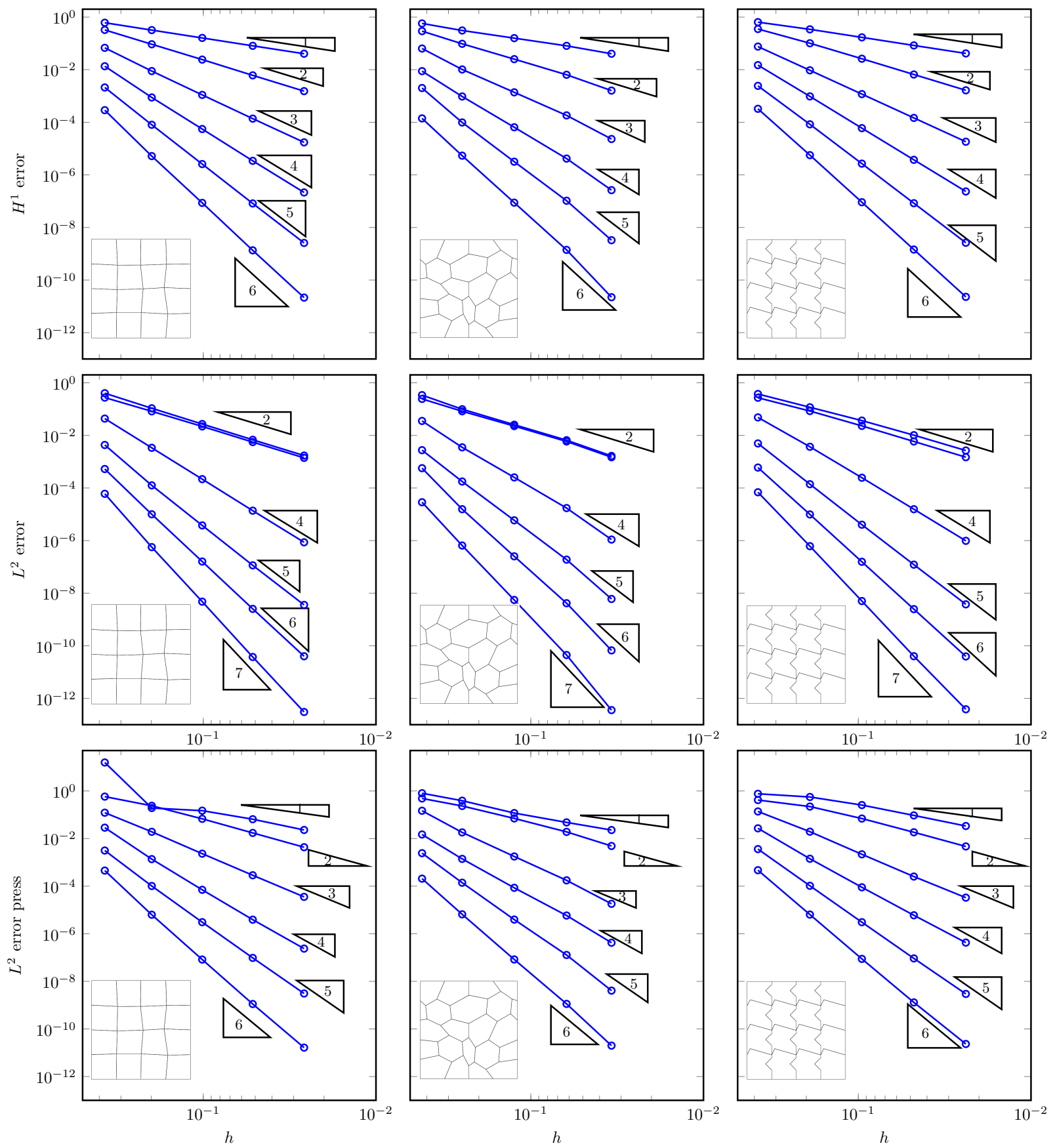

Fig. 4. Convergence curves versus the mesh size parameter $h$ for the velocity approximation measured using the energy norm (55) (top panels) and the $L^{2}$-norm (56) (mid panels), and for the pressure approximation measured using the $L^{2}$-norm (57) (bottom panels). Blue lines with circles represent the error curves for the Basic Formulation using the virtual element space (13). The right-hand side is approximated by using the projection operator $\Pi_{k-2}^{0}$. The mesh families used in each calculations are shown in the left corner of each panel. The expected convergence slopes and rates are shown by the triangles and corresponding numeric labels. 

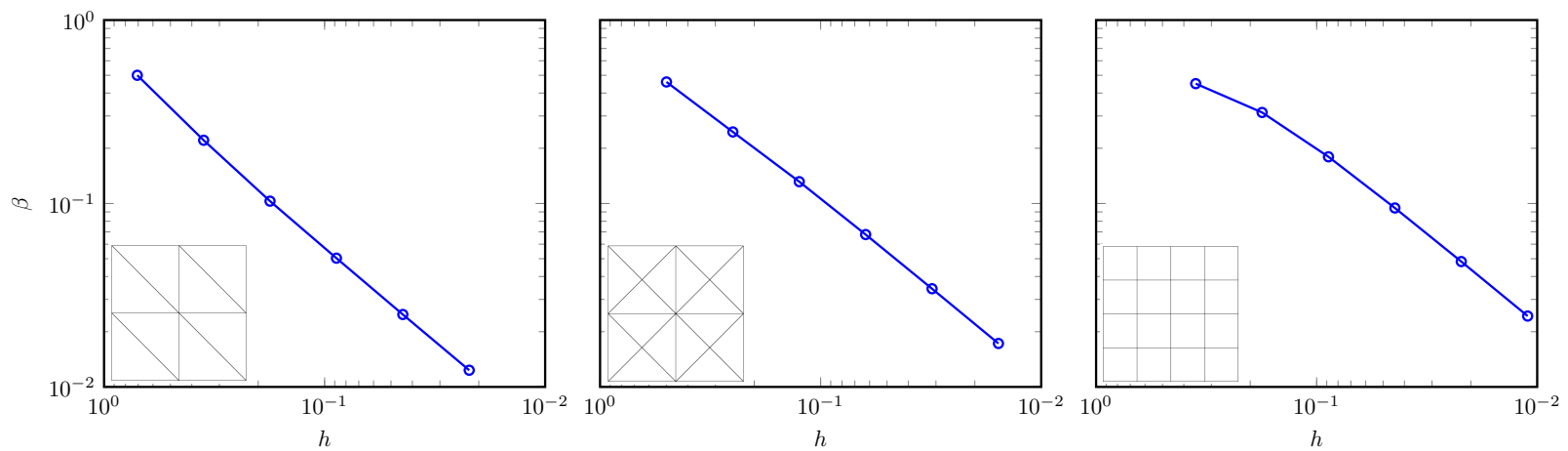

Fig. 5. Values of the inf-sup constant $\beta$ versus the mesh size parameter $h$. Blue lines with circles represent the error curves for the Basic Formulation using the virtual element space (13). The mesh families used in each calculations are shown in the bottom-left corner of each panel.

\section{Acknowledgments}

Dr. G. Manzini was supported by the LDRD-ER program of Los Alamos National Laboratory under project number 20180428ER. Los Alamos National Laboratory is operated by Triad National Security, LLC, for the National Nuclear Security Administration of U.S. Department of Energy (Contract No. 89233218CNA000001).

\section{References}

[1] R. A. Adams and J. J. F. Fournier. Sobolev spaces. Pure and Applied Mathematics. Academic Press, 2 edition, 2003.

[2] B. Ahmad, A. Alsaedi, F. Brezzi, L. D. Marini, and A. Russo. Equivalent projectors for virtual element methods. Comput. Math. Appl., 66:376-391, September 2013.

[3] P. F. Antonietti, L. Beirão da Veiga, D. Mora, and M. Verani. A stream virtual element formulation of the Stokes problem on polygonal meshes. SIAM J. Numer. Anal., 52(1):386-404, 2014.

[4] P. F. Antonietti, G. Manzini, and M. Verani. The fully nonconforming Virtual Element method for biharmonic problems. Math. Models Methods Appl. Sci., 28(2), 2018.

[5] P. F. Antonietti, G. Manzini, and M. Verani. The conforming virtual element method for polyharmonic problems. Comput. Math. Appl., 79(7):2021-2034, 2020. published online: 4 October 2019.

[6] B. Ayuso de Dios, K. Lipnikov, and G. Manzini. The non-conforming virtual element method. ESAIM Math. Model. Numer., 50(3):879-904, 2016.

[7] B. Bang and D. Lukkassen. Application of homogenization theory related to Stokes flow in porous media. Appl. Math., 44:309-319, 1999.

[8] L. Beirão da Veiga, F. Brezzi, A. Cangiani, G. Manzini, L. D. Marini, and A. Russo. Basic principles of virtual element methods. Math. Models Methods Appl. Sci., 23:119-214, 2013.

[9] L. Beirão da Veiga, F. Brezzi, and L. D. Marini. Virtual elements for linear elasticity problems. SIAM J. Numer. Anal., 51(2):794-812, 2013.

[10] L. Beirão da Veiga, F. Brezzi, L. D. Marini, and A. Russo. The hitchhiker's guide to the virtual element method. Math. Models Methods Appl. Sci, 24(8):1541-1573, 2014.

[11] L. Beirão da Veiga, F. Brezzi, L. D. Marini, and A. Russo. Virtual element methods for general second order elliptic problems on polygonal meshes. Math. Models Methods Appl. Sci., 26(04):729-750, 2015.

[12] L. Beirão da Veiga, F. Brezzi, L. D. Marini, and A. Russo. H(div) and H(curl)-conforming VEM. Numer. Math., 133(2):303-332, 2016.

[13] L. Beirão da Veiga, F. Brezzi, L. D. Marini, and A. Russo. Mixed virtual element methods for general second order elliptic problems on polygonal meshes. ESAIM Math. Model. Num., 50(3):727-747, 2016.

[14] L. Beirão da Veiga, F. Brezzi, L. D. Marini, and A. Russo. Serendipity nodal VEM spaces. Comput. Fluids, 141:2-12, 2016.

[15] L. Beirão da Veiga, F. Brezzi, L. D. Marini, and A. Russo. Virtual element methods for general second order elliptic problems on polygonal meshes. Math. Models Methods Appl. Sci., 26(4):729-750, 2016.

[16] L. Beirão da Veiga, A. Chernov, L. Mascotto, and A. Russo. Basic principles of hp virtual elements on quasiuniform meshes. Math. Models Methods Appl. Sci., 26(8):1567-1598, 2016.

[17] L. Beirão da Veiga, F. Dassi, and G. Vacca. The Stokes complex for virtual elements in three dimensions. Math. Models Methods Appl. Sci., 30(03):477-512, 2020.

[18] L. Beirão da Veiga, V. Gyrya, K. Lipnikov, and G. Manzini. Mimetic finite difference method for the Stokes problem on polygonal meshes. J. Comput. Phys., 228:7215-7232, 2009.

[19] L. Beirão da Veiga and K. Lipnikov. A mimetic discretization of the Stokes problem with selected edge bubbles. SIAM J. Sci. Comput., 32(2):875-893, 2010. 

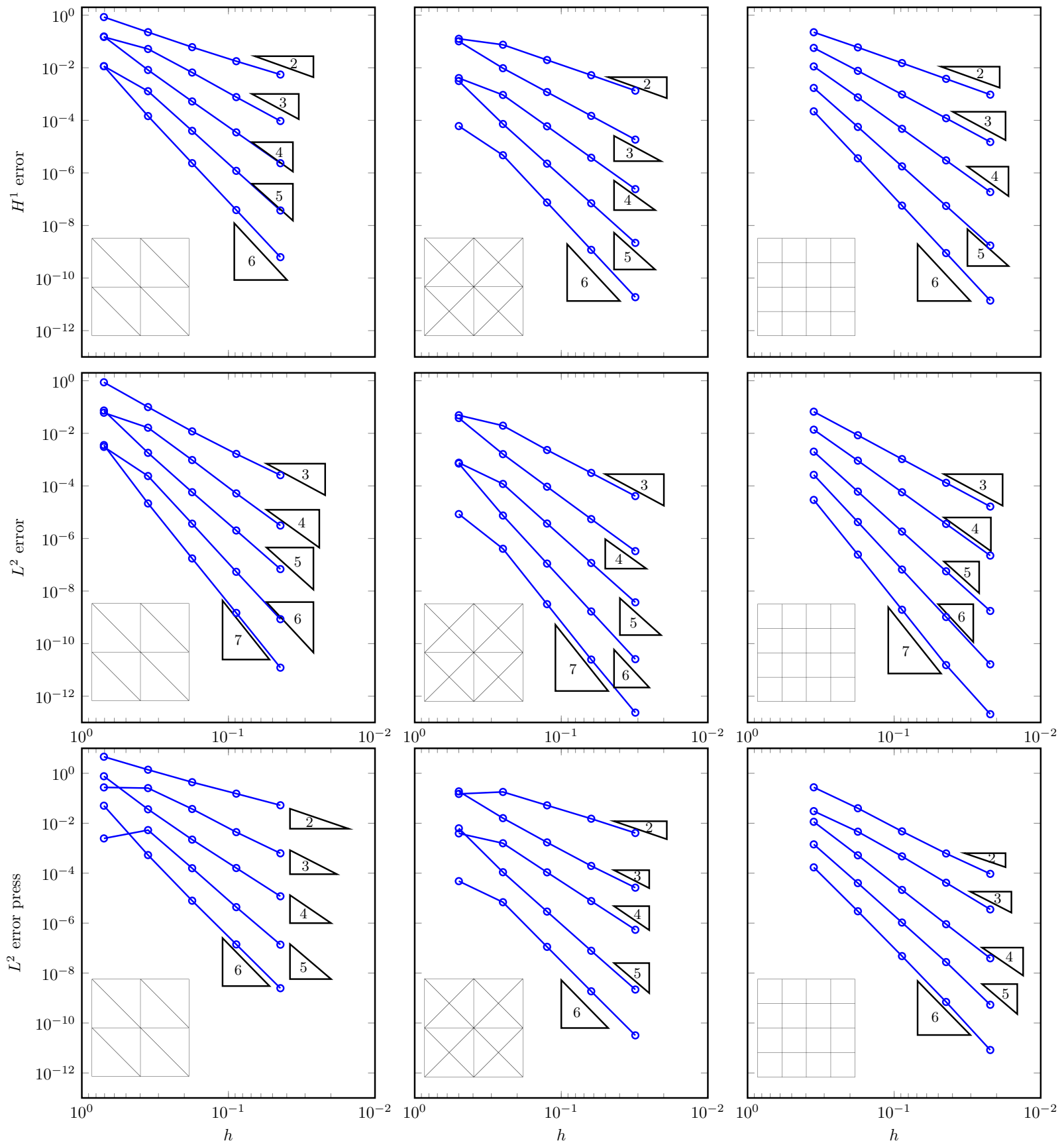

Fig. 6. Convergence curves versus the mesh size parameter $h$ for the velocity approximation measured using the energy norm (55) (top panels) and the $L^{2}$-norm (56) (mid panels), and for the pressure approximation measured using the $L^{2}$-norm (57) (bottom panels). Blue lines with circles represent the error curves for the Basic Formulation using the enhanced virtual element space (14). The right-hand side is approximated by using the projection operator $\Pi_{k}^{0}$. The mesh families used in each calculations are shown in the left corner of each panel. The results with $k=1$ are not reported because there is no convergence. The expected convergence slopes and rates are shown by the triangles and corresponding numeric labels. 

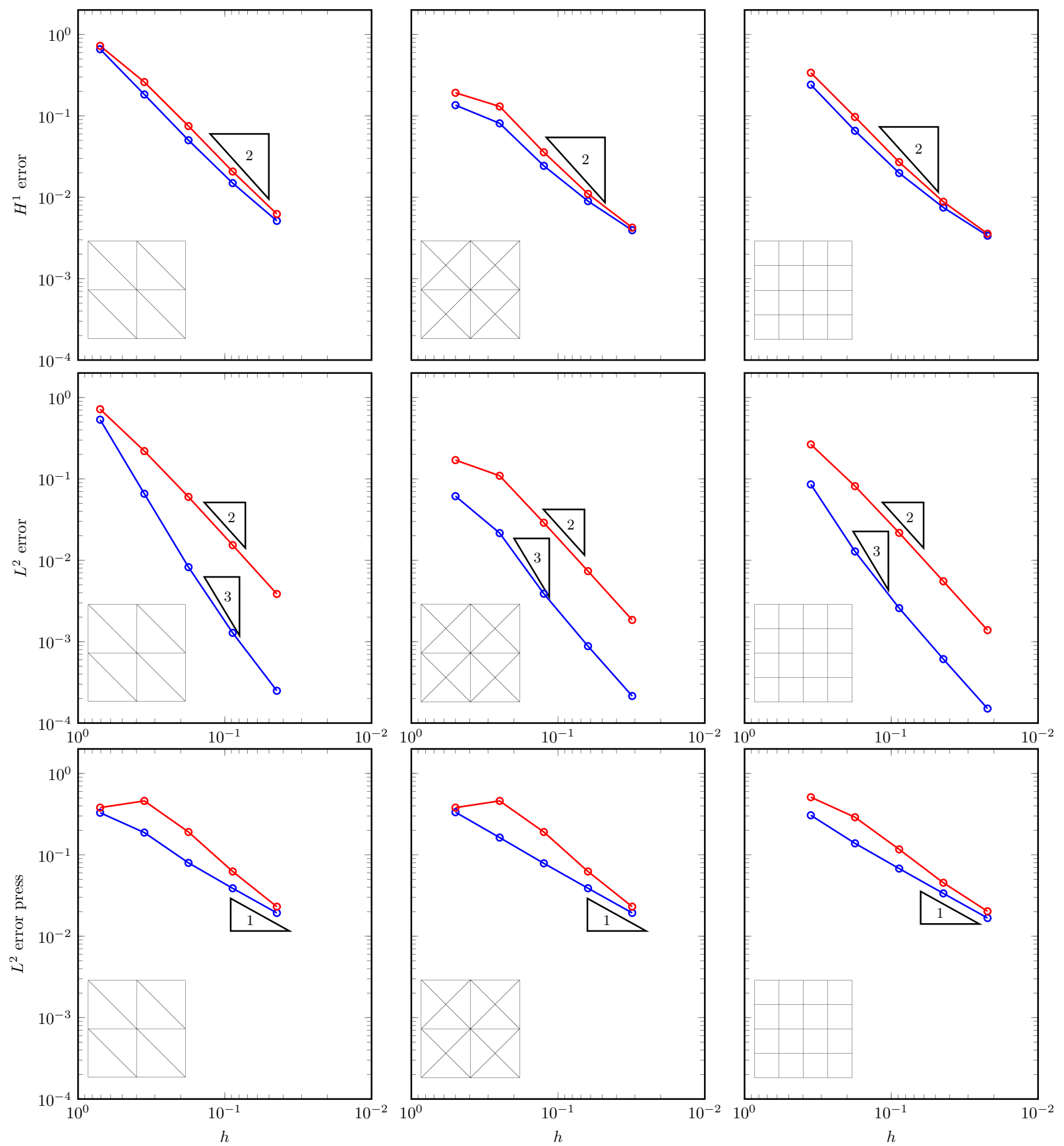

Fig. 7. Convergence curves versus the mesh size parameter $h$ for the velocity approximation measured using the energy norm (55) (top panels) and the $L^{2}$-norm (56) (mid panels), and for the pressure approximation measured using the $L^{2}$-norm (57) (bottom panels). Blue lines with circles represent the error curves for the formulation using the enhanced virtual element space (14) with the right-hand side approximated by using the projection operator $\Pi_{k}^{0}$. Red lines with circles represent the error curve with the right-hand side approximated by using the projection operator $\Pi_{k-2}^{0}$. The mesh families used in each calculations are shown in the left corner of each panel. The convergence slopes and rates are shown by the triangles and corresponding numeric labels. 
[20] L. Beirão da Veiga, K. Lipnikov, and G. Manzini. Error analysis for a mimetic discretization of the steady Stokes problem on polyhedral meshes. SIAM J. Numer. Anal., 48:1419-1443, 2010.

[21] L. Beirão da Veiga, K. Lipnikov, and G. Manzini. The Mimetic Finite Difference Method, volume 11 of MS\&A. Modeling, Simulations and Applications. Springer, I edition, 2014.

[22] L. Beirão da Veiga, C. Lovadina, and D. Mora. A virtual element method for elastic and inelastic problems on polytope meshes. Comput. Methods Appl. Mech. Engrg., 295:327-346, 2015.

[23] L. Beirão da Veiga, C. Lovadina, and G. Vacca. Divergence free virtual elements for the Stokes problem on polygonal meshes. ESAIM Math Model. Numer, 51(2):509-535, 2017.

[24] L. Beirão da Veiga, C. Lovadina, and G. Vacca. Virtual elements for the Navier-Stokes problem on polygonal meshes. SIAM J. Numer. Anal., 56(3):1210-1242, 2018.

[25] L. Beirão da Veiga and G. Manzini. A virtual element method with arbitrary regularity. IMA J. Numer. Anal., 34(2):782-799, 2014.

[26] L. Beirão da Veiga and G. Manzini. Residual a posteriori error estimation for the virtual element method for elliptic problems. ESAIM Math. Model. Numer., 49:577-599, 2015.

[27] L. Beirão da Veiga, G. Manzini, and L. Mascotto. A posteriori error estimation and adaptivity in hp virtual elements. Numer. Math., 143:139$175,2019$.

[28] L. Beirão da Veiga, D. Mora, and G. Vacca. The Stokes complex for virtual elements with application to Navier-Stokes flows. J. Sci. Comput., 81:990-1018, 2019.

[29] M. F. Benedetto, S. Berrone, and A. Borio. The Virtual Element Method for underground flow simulations in fractured media. In Advances in Discretization Methods, volume 12 of SEMA SIMAI Springer Series, pages 167-186. Springer International Publishing, Switzerland, 2016.

[30] M. F. Benedetto, S. Berrone, S. Pieraccini, and S. Scialò. The virtual element method for discrete fracture network simulations. Comput. Methods Appl. Mech. Engrg., 280(0):135 - 156, 2014.

[31] E. Benvenuti, A. Chiozzi, G. Manzini, and N. Sukumar. Extended virtual element method for the Laplace problem with singularities and discontinuities. Comput. Methods Appl. Mech. Engrg., 356:571 - 597, 2019.

[32] S. Berrone and A. Borio. Orthogonal polynomials in badly shaped polygonal elements for the Virtual Element Method. Finite Elements in Analysis \& Design, 129:14-31, 2017.

[33] S. Berrone, A. Borio, and Manzini. SUPG stabilization for the nonconforming virtual element method for advection-diffusion-reaction equations. Comput. Methods Appl. Mech. Engrg., 340:500-529, 2018.

[34] S. Berrone, A. Borio, and S. Scialò. A posteriori error estimate for a PDE-constrained optimization formulation for the flow in DFNs. SIAM J. Numer. Anal., 54(1):242-261, 2016.

[35] S. Berrone, S. Pieraccini, and S. Scialò. Towards effective flow simulations in realistic discrete fracture networks. J. Comput. Phys., 310:181201, 2016.

[36] S. Berrone, S. Pieraccini, S. Scialò, and F. Vicini. A parallel solver for large scale DFN flow simulations. SIAM J. Sci. Comput., 37(3):C285C306, 2015

[37] D. Boffi, F. Brezzi, and M. Fortin. Mixed finite element methods and applications, volume 44. Springer, 2013.

[38] S. C. Brenner and L. R. Scott. The mathematical theory of finite element methods, volume 15 of Texts in Applied Mathematics. Springer-Verlag, New York, 1994.

[39] F. Brezzi, R. S. Falk, and L. D. Marini. Basic principles of mixed virtual element methods. ESAIM Math. Model. Numer. Anal., 48(4):12271240, 2014.

[40] F. Brezzi, K. Lipnikov, and M. Shashkov. Convergence of mimetic finite difference method for diffusion problems on polyhedral meshes with curved faces. Math. Models Methods Appl. Sci., 16(2):275-297, 2006.

[41] F. Brezzi, K. Lipnikov, M. Shashkov, and V. Simoncini. A new discretization methodology for diffusion problems on generalized polyhedral meshes. Comput. Methods Appl. Mech. Engrg., 196(37-40):3682-3692, 2007.

[42] F. Brezzi and L. D. Marini. Virtual element methods for plate bending problems. Comput. Methods Appl. Mech. Engrg., 253:455-462, 2013.

[43] Z. Cai, C. Tong, P. S. Vassilevski, and C. Wang. Mixed finite element methods for incompressible flow: stationary Stokes equations. Numer Methods Partial Differ. Equ., 26:957-978, 2010.

[44] J. Campbell and M. Shashkov. A tensor artificial viscosity using a mimetic finite difference algorithm. J. Comput. Phys., 172:739-765, 2001.

[45] A. Cangiani, E. H. Georgoulis, T. Pryer, and O. J. Sutton. A posteriori error estimates for the virtual element method. Numer. Math., pages $1-37,2017$.

[46] A. Cangiani, V. Gyrya, and G. Manzini. The non-conforming virtual element method for the Stokes equations. SIAM J. Numer. Anal., 54(6):3411-3435, 2016.

[47] A. Cangiani, V. Gyya, G. Manzini, and Sutton. O. Chapter 14: Virtual element methods for elliptic problems on polygonal meshes. In K. Hormann and N. Sukumar, editors, Generalized Barycentric Coordinates in Computer Graphics and Computational Mechanics, pages 1-20. CRC Press, Taylor \& Francis Group, 2017.

[48] A. Cangiani, G. Manzini, and A. Russo. Convergence analysis of a mimetic finite difference method for elliptic problems. SIAM J. Numer. Anal., 47(4):2612-2637, 2009.

[49] A. Cangiani, G. Manzini, A. Russo, and N. Sukumar. Hourglass stabilization of the virtual element method. Int. J. Numer. Methods Eng., 102(3-4):404-436, 2015.

[50] A. Cangiani, G. Manzini, and O. Sutton. Conforming and nonconforming virtual element methods for elliptic problems. IMA J. Numer. Anal., 37:1317-1354, 2017. (online August 2016).

[51] O. Certik, F. Gardini, G. Manzini, L. Mascotto, and G. Vacca. The p- and hp-versions of the virtual element method for elliptic eigenvalue problems. Comput. Math. Appl., 2019. published online: 31 October 2019.

[52] O. Certik, F. Gardini, G. Manzini, and G. Vacca. The virtual element method for eigenvalue problems with potential terms on polytopic meshes. Appl. Math., 63(3):333-365, 2018. 
[53] A. Chernov, C. Marcati, and L. Mascotto. p- and hp- virtual elements for the stokes problem. Adv. Comput. Math., 2021. published online: 23 March 2021.

[54] M. Crouzeix and P. A. Raviart. Conforming and nonconforming finite element methods for solving the stationary Stokes equations. RAIRO Ser. Rouge, 7:33-75, 1973.

[55] D. A. Di Pietro, J. Droniou, and G. Manzini. Discontinuous skeletal gradient discretisation methods on polytopal meshes. J. Comput. Phys., 355:397-425, 2018.

[56] F. Gardini, G. Manzini, and G. Vacca. The nonconforming virtual element method for eigenvalue problems. ESAIM Math. Model. Numer., 53:749-774, 2019. Accepted for publication: 29 November 2018. DOI: 10.1051/m2an/2018074.

[57] V. Girault and P.-A. Raviart. Finite Element Approximation of the Navier-Stokes Equations, volume 749 of Lecture Notes in Mathematics. Springer-Verlag, Berlin, Heidelberg, 1 edition, 1979.

[58] V. Girault and P.-A. Raviart. Finite Element Methods for Navier-Stokes Equations: Theory and Algorithms. Springer Series in Computational Mathematics. Springer-Verlag, 1986.

[59] R. M. Höfer. Sedimentation of inertialess particles in Stokes flows. Commun. Math. Phys., 360:55-10, 2018.

[60] J. Hyman and M. Shashkov. Mimetic discretizations for Maxwell's equations and the equations of magnetic diffusion. PIER, 32:89-121, 2001.

[61] H. Kitahata, N. Yoshinaga, K. H. Nagai, and Y. Sumino. 3 - Dynamics of Droplets. In S. Kinoshita, editor, Pattern Formations and Oscillatory Phenomena, pages 85-118. Elsevier, Boston, 2013.

[62] Y. Kuznetsov and S. Repin. New mixed finite element method on polygonal and polyhedral meshes. Russian J. Numer. Anal. Math. Modelling, 18:261-278, 2003.

[63] S. Linden, L. Cheng, and A. Wiegmann. Specialized methods for direct numerical simulations in porous media. Technical Report Report M2M-2018-01, Math2Market GmbH, Kaiserslautern, Germany, October 2018.

[64] K. Lipnikov, G. Manzini, and M. Shashkov. Mimetic finite difference method. J. Comput. Phys., 257 - Part B:1163-1227, 2014. Review paper.

[65] G. Manzini, A. Russo, and N. Sukumar. New perspectives on polygonal and polyhedral finite element methods. Math. Models Methods Appl. Sci, 24(8):1621-1663, 2014.

[66] L. Mascotto. Ill-conditioning in the virtual element method: stabilizations and bases. Numer. Methods Partial Differ. Equ., 34(4):1258-1281, 2018.

[67] D. Mora, G. Rivera, and R. Rodríguez. A virtual element method for the Steklov eigenvalue problem. Math. Models Methods Appl. Sci., 25(08):1421-1445, 2015.

[68] S. Natarajan, P. A. Bordas, and E. T. Ooi. Virtual and smoothed finite elements: a connection and its application to polygonal/polyhedral finite element methods. Int. J. Numer. Methods Engrg., 104(13):1173-1199, 2015.

[69] G. H. Paulino and A. L. Gain. Bridging art and engineering using Escher-based virtual elements. Struct. and Multidisciplinary Optim., 51(4):867-883, 2015

[70] I. Perugia, P. Pietra, and A. Russo. A plane wave virtual element method for the Helmholtz problem. ESAIM Math. Model. Num., 50(3):783$808,2016$.

[71] Jinshui Qin. On the convergence of some low order mixed finite elements for incompressible fluids. The Pennsylvania State University, 1994. Thesis.

[72] J. P. Smith, A. C. Barbati, S. M. Santana, J. P. Gleghorn, and B. J. Kirby. Microfluidic transport in microdevices for rare cell capture. Electrophoresis, 33(21):3133-3142, 2012.

[73] N. Sukumar and A. Tabarraei. Conforming polygonal finite elements. Int. J. Numer. Methods Eng., 61:2045-2066, 2004.

[74] A. Tabarraei and N. Sukumar. Extended finite element method on polygonal and quadtree meshes. Comput. Methods Appl. Mech. Engrg., 197:425-438, 2007.

[75] C. Talischi, G. H. Paulino, A. Pereira, and I. F. M. Menezes. Polygonal finite elements for topology optimization: A unifying paradigm. Int. J. Numer. Methods Eng., 82:671-698, 2010.

[76] G. Vacca and L. Beirão da Veiga. Virtual element methods for parabolic problems on polygonal meshes. Numer. Methods for PDEs, 31(6):2110-2134, 2015.

[77] E. Wachspress. Rational Bases and Generalized Barycentrics: Applications to Finite Elements and Graphics. Technology \& Engineering. Springer, 2015.

[78] P. Wriggers, W. T. Rust, and B. D. Reddy. A virtual element method for contact. Comput. Mech., 58(6):1039-1050, 2016.

[79] J. Zhao, S. Chen, and B. Zhang. The nonconforming virtual element method for plate bending problems. Math. Models Methods Appl. Sci., 26(9):1671-1687, 2016. 


\section{Appendix A. Approximation and orthogonality results for the virtual element method}

In this appendix, we report three technical lemmas that we use in the convergence analysis of Section 4 . The first two lemmas state basic results for the approximation of a vector-valued field by its virtual element interpolant and a scalar function by its polynomial projection onto the subspace of polynomials, and are presented without a proof. The interpolants $\mathbf{v}_{I}$ and $q_{I}$ are an approximation of $\mathbf{v}$ and $q$, and the interpolation error can be bounded as stated in the following lemma. The third lemma presents an identity that is used in the proof of Theorem 4.8.

Lemma A.1 (Projection error $[\mathbf{8 , 3 8}]$ ) Under Assumptions (M1)-(M2), for every vector-valued field $\mathbf{v} \in\left[H^{s+1}(E)\right]^{2}$ and scalar function $q \in H^{s}(E)$ with $1 \leq s \leq \ell$, there exists a vector polynomial $\mathbf{v}_{\pi} \in\left[\mathbb{P}_{\ell}(E)\right]^{2}$ and a scalar polynomial $q_{\pi} \in \mathbb{P}_{\ell}(E)$ such that

$$
\begin{aligned}
& \left\|\mathbf{v}-\mathbf{v}_{\pi}\right\|_{0, E}+h_{E}\left|\mathbf{v}-\mathbf{v}_{\pi}\right|_{1, E} \leq C h_{E}^{s+1}|\mathbf{v}|_{s+1, E}, \\
& \| q-q_{\pi}||_{0, E}+h_{E}\left|q-q_{\pi}\right|_{1, E} \leq C h_{E}^{s}|q|_{s, E}
\end{aligned}
$$

for some positive constant $C$ that is independent of $h_{E}$ but may depend on the polynomial degree $\ell$ and the mesh regularity constant $\varrho$.

Lemma A.2 (Interpolation error $[\mathbf{8 , 3 8}]$ ) Under Assumptions (M1)-(M2), for every vector-valued field $\mathbf{v} \in\left[H^{s+1}(E)\right]^{2}$ and scalar function $q \in H^{s}(E)$ with $1 \leq s \leq \ell$, there exists a vector valued-field $\mathbf{v}_{I} \in \mathbf{V}_{k}^{h}(E)$ and a scalar field $q \in Q_{\ell}^{h}$ such that

$$
\begin{array}{r}
\| \mathbf{v}-\mathbf{v}_{I}||_{0, E}+h_{E}\left|\mathbf{v}-\mathbf{v}_{I}\right|_{1, E} \leq C h_{E}^{s+1}|\mathbf{v}|_{s+1, E}, \\
\left\|q-q_{I}\right\|_{0, E}+h_{E}\left|q-q_{I}\right|_{1, E} \leq C h_{E}^{s}|q|_{s, E}
\end{array}
$$

for some positive constant $C$ that is independent of $h_{E}$ but may depend on the polynomial degree $\ell$ and the mesh regularity constant $\varrho$.

Lemma A.3 (Orthogonality between $\operatorname{div}\left(\mathbf{u}_{h}-\mathbf{u}_{I}\right)$ and $\left.p_{h}-p_{I}\right)$ Let $\mathbf{u} \in\left[H_{0}^{1}(\Omega)\right]^{2}$ be the exact solution of the variational formulation of the Stokes problem given in (7)-(8). Let $\left(\mathbf{u}_{h}, p_{h}\right) \in \mathbf{V}_{k}^{h} \times Q_{k-1}^{h}$ be the solution of the virtual element approximation (10)-(11). Then, it holds that

$$
b\left(\mathbf{u}_{h}-\mathbf{u}_{I}, p_{h}-p_{I}\right)=0 \quad \forall q \in Q^{h} .
$$

Proof. First, note that the composed operator $\Pi_{k-1}^{0, \mathrm{E}} \operatorname{div}(\cdot)$ only depends on the degrees of freedom of its argument. These degrees of freedom are the same for $\mathbf{u}$ and its virtual element interpolation $\mathbf{u}_{I}$, so that it must hold that $\Pi_{k-1}^{0, \mathrm{E}} \operatorname{div} \mathbf{u}=\Pi_{k-1}^{0, \mathrm{E}} \operatorname{div} \mathbf{u}_{I}$. Using this property and the definition of the orthogonal projection $\Pi_{k-1}^{0, \mathrm{E}}$ yield

$$
\begin{aligned}
b(\mathbf{u}, q) & =\sum_{\mathrm{E}} \int_{\mathrm{E}} q \operatorname{div} \mathbf{u} d \mathbf{x}=\sum_{\mathrm{E}} \int_{\mathrm{E}} q \Pi_{k-1}^{0, \mathrm{E}} \operatorname{div} \mathbf{u} d \mathbf{x}=\sum_{\mathrm{E}} \int_{\mathrm{E}} q \Pi_{k-1}^{0, \mathrm{E}} \operatorname{div} \mathbf{u}_{I} d \mathbf{x} \\
& =\sum_{\mathrm{E}} \int_{\mathrm{E}} q \operatorname{div} \mathbf{u}_{I} d \mathbf{x}=b\left(\mathbf{u}_{I}, q\right)
\end{aligned}
$$

for every $q \in \mathbb{P}_{k-1}(\mathrm{E})$. Eventually, we note that $0=b(\mathbf{u}, q)=b\left(\mathbf{u}_{I}, q\right)$ from Eq. (8) and $b\left(\mathbf{u}_{h}, q\right)=b_{h}\left(\mathbf{u}_{h}, q\right)$ from Eq. (33). So, it holds that $b\left(\mathbf{u}_{h}-\mathbf{u}_{I}, q\right)=0$, and relation (A.5) immediately follows by setting $q=p_{h}-p_{I}$. 